\title{
Fauna silvestre con valor de uso en la Reserva de la Biosfera Sierra de Huautla, Morelos, México
}

\author{
Wild fauna with use value in the Biosphere Reserve Sierra de Huautla, Morelos, Mexico
}

Raúl Valle Marquina ${ }^{1}$

https://orcid.org/0000-0003-4730-2973

rvallemarquina@gmail.com

\section{Alejandro García Flores* 2 \\ http://orcid.org/0000-0002-1122-5059 \\ alejandro.garcia@uaem.mx \\ Hortensia Colín Bahena ${ }^{2}$ \\ https://orcid.org/0000-0001-5248-9846 \\ ortencia.colin@uaem.mx \\ *Corresponding author}

1 Programa de Maestría en Manejo de Recursos Naturales, Centro de Investigaciones Biológicas, Universidad Autónoma del Estado de Morelos, Av. Universidad \#1001, Chamilpa, Cuernavaca, Morelos, C. P. 62209, México.

2 Centro de Investigaciones Biológicas, Universidad Autónoma del Estado de Morelos, Av. Universidad \#1001, Chamilpa, Cuernavaca, Morelos, C. P. 62209, México.

\section{Citación}

Valle Marquina R, García Flores A, Colín Bahena H. 2021. Fauna silvestre con valor de uso en la Reserva de la Biosfera Sierra de Huautla, Morelos, México. Revista peruana de biología 28(4): e19921 001- 016 (Noviembre 2021). doi: http://dx.doi.org/10.15381/rpb v28i4.19921

$\begin{array}{ll}\text { Presentado: } & 06 / 05 / 2021 \\ \text { Aceptado: } & 22 / 08 / 2021 \\ \text { Publicado online: } & 26 / 11 / 2021\end{array}$

Editor:

Diana Silva

\section{Resumen}

El objetivo de este trabajo fue sistematizar las especies apropiadas, formas de apropiación y valores de uso asignados a la fauna silvestre en comunidades de la Reserva de la Biosfera Sierra de Huautla, México, mediante un análisis documental y trabajo de campo. Se registraron 46 especies con valor de uso pertenecientes a 31 familias. La clase con mayor número de especies apropiadas son las aves (42.5\%), seguidas de los mamíferos (34\%). El 64\% de las especies se apropian con fines alimentarios y el $38.3 \%$ se utilizan para el tratamiento de enfermedades en la medicina tradicional. Otros usos son adornos, amuletos, elaboración de herramientas y como animal de compañía. La apropiación de fauna silvestre se realiza por medio de la cacería, la recolección y la pesca. Las armas de fuego como la escopeta es el principal instrumento para la cacería. También se utilizan perros, machetes, resorteras, tarrayas, anzuelos, nudos corredizos y se capturan manualmente. La apropiación puede ser una actividad oportunista $\mathrm{u}$ organizada por medio de las arreadas, espiadas, lampareadas, campeadas, acorralamiento y recolección manual. La apropiación de fauna silvestre es una actividad culturalmente arraigada en las comunidades de la reserva. Sin embargo, el análisis de la relación sociedad-fauna en la región es limitado, por lo que es necesario generar información que promueva el manejo sostenible del recurso faunístico.

\begin{abstract}
The aims of this paper were the systematization of appropriated species, appropriation forms and assigned use values of wildlife species in the Biosphere Reserve of the Sierra de Huautla, Mexico, by means of documentary analysis and field work. We recorded 46 species with use value pertaining to 31 families. Birds accounted for the highest number of appropriated species (42.5\%), followed by mammals (34\%). Of the total species, $64 \%$ are appropriated for alimentary purposes and are used in the treatment of diseases in traditional medicine. Others are used as ornaments, talismans, in tool manufacture and as pet animals. Wildlife appropriation is achieved by hunting, collection and fishing. Fire arms such as shotguns are the main instrument used in hunting. Dogs, machetes, slingshots, nets, hooks, slipknots, are also used, or they are captured by hand. Appropriation can be an opportunistic or organized activity by means of "arreadas" (herding), "espiadas" (opportunistic observation), "lampareadas" (spotlighting), "campeadas" (scouring), "acorralamiento" (cornering) and manual collection. Wildlife appropriation is a culturally rooted activity in the reserve communities. Nevertheless, the analysis of the relationship society-fauna in the region is limited and this is why the generation of information that may enable a sustainable management of the faunistic resource is necessary.
\end{abstract}

Palabras clave:

Apropiación de la naturaleza; cacería; valor de uso; selva baja caducifolia; Sierra de Huautla; fauna silvestre; Áreas Naturales Protegidas; etnobiología.

Keywords:

Appropriation of nature; hunting; use value; low deciduous forest; Sierra de Huautla; Wildlife; protected areas; ethnobiology. 


\section{Introducción}

En la relación histórica sociedad-naturaleza, las comunidades indígenas y campesinas han generado conocimientos ecológicos tradicionales y prácticas locales que les ha permitido la apropiación de los recursos naturales dentro de sus territorios, de acuerdo con su propia cosmovisión (Toledo et al. 2019). La fauna silvestre representa un recurso con el que estos grupos tradicionales han establecido múltiples interacciones, desde su aporte de bienes para cubrir diversas necesidades materiales, hasta su papel simbólico en su cosmogonía y cosmovisión; aspectos que varían de acuerdo con cada contexto cultural, histórico y geográfico (Pérez-Gil et al. 1995, Alves $\&$ Albuquerque 2018).

En México, los animales silvestres constituyen parte significativa de la realidad y cotidianidad de diversos grupos culturales desde la época prehispánica (Argueta 2008). Estudios arqueozoológicos y fuentes históricas demuestran que las culturas precolombinas aprovechaban diferentes especies de fauna silvestre para satisfacer necesidades de alimento, medicina, abrigo, vestimenta, obtención de fibras, herramientas o como objetos de intercambio en los mercados prehispánicos (De Sahagún 2005, Götz 2014, Valadez-Azúa \& Rodríguez-Galicia 2014). También representaron elementos importantes de su cosmovisión, al personificar dioses o figuras centrales en rituales (Olivier 2015).

En los patrones actuales de aprovechamiento, la extracción de fauna mediante la cacería forma parte de las estrategias de subsistencia y manejo de los recursos naturales en comunidades campesinas e indígenas, particularmente de aquellas que habitan cerca de áreas forestales. La cacería es una práctica tradicional con importancia sociocultural que provee principalmente alimentos, pero también productos para la medicina tradicional, ornamentos, fabricación de herramientas, animales de compañía o para la obtención de ingresos económicos, como lo evidencian diferentes estudios en el país (Santos-Fita et al. 2012, Ramírez-Barajas \& Calme 2015, Herrera-Flores et al. 2019, Solís \& Casas 2019, Zarazúa-Carbajal et al. 2020). Análisis bibliográficos a nivel nacional muestran que existe una amplia diversidad faunística con valores de uso. Se registran hasta 82 especies de mamíferos silvestres aprovechados principalmente con fines alimentarios o utilizados con fines medicinales (Ávila-Nájera et al. 2018a). Así mismo, se documentan 103 reptiles y 32 anfibios que tienen al menos un valor de uso (Ávila-Nájera et al. 2018b). En la práctica de la medicina tradicional mexicana, revisiones han contabilizado hasta 163 especies animales empleados con fines terapéuticos (Alonso-Castro 2014).

La cacería en su complejo escenario sociocultural contemporáneo, no solo es un medio de subsistencia. Su importancia multidimensional también la concibe como una práctica formadora de relaciones sociales, además como una actividad simbólica donde se expresa la cosmovisión de los grupos originarios (Plata et al. 2019, Méndez-Sánchez 2020). Ante estas premisas, el objetivo del presente trabajo fue sistematizar mediante el análisis de literatura y trabajo en campo el número de especies apropiadas, formas de apropiación y valores de uso de la fauna silvestre en Sierra de Huautla, Morelos, México.

\section{Material y métodos}

Área de estudio. - La Sierra de Huautla se ubica en la parte sur del estado de Morelos en el centro de México (Figura 1). Limita con el estado de Guerrero y Puebla dentro de la Cuenca Alta del Balsas, región relevante por sus características ecológicas e importancia sociocultural. En 1999 fue decretada como Área Natural Protegida en la modalidad de Reserva de la Biosfera (REBIOSH), por ser un reservorio de biodiversidad del trópico seco en México (Dorado et al. 2002). La REBIOSH conserva uno de los ecosistemas con mayor vulnerabilidad para su preservación en el país como la selva baja caducifolia. Para el estado de Morelos representa la mayor superficie de este tipo de vegetación. En la zona también se distribuyen otros tipos de vegetación como el bosque de encino, de pino y selva mediana subcaducifolia, aunque en menor superficie (Dorado et al. 2002).

En las 59030.94 hectáreas de superficie (de las cuales 48798. 84 se encuentra en Morelos), se distribuye una amplia diversidad biológica. Estudios florísticos registran 1035 especies de plantas vasculares, distribuidas en 511 géneros y 132 familias botánicas (Arias et al. 2014). Los estudios faunísticos, aunque solo han incluido ciertos grupos en determinadas zonas, reportan 8 especies de peces, 17 de anfibios, 53 de reptiles, 200 de aves y 72 especies de mamíferos (Valenzuela et al. 2010). La riqueza de invertebrados está distribuida en 292 especies de mariposas diurnas, 50 de odonatos, 230 de abejas y hasta 1,480 especies de coleópteros (Valenzuela et al. 2010, Toledo et al. 2012).

En el contexto social, en la REBIOSH se encuentran 30 comunidades que pertenecen a los municipios de Amacuzac, Puente de Ixtla, Jojutla, Tlaquiltenango y Tepalcingo (DOF 1999) (Fig. 1). La población total es de 19383 habitantes de acuerdo con datos Instituto Nacional de Estadística y Geografía (INEGI 2010).

Los vestigios arqueológicos registrados en comunidades como Chimalacatlan, Coaxitlán, Huaxtla, demuestran que la región ha sido habitada desde la época prehispánica (Durand 2010). El 90.32\% de las localidades se clasifican con alta marginación, el $6.45 \%$ con grado medio y el $3.22 \%$ con grado muy alto (INEGI 2010). Los altos índices de marginación se traducen en falta de servicios básicos, oportunidades de educación, salud y empleo, escenario por el cual se considera como la región con mayor marginación social de Morelos (CONABIO \& UAEM 2004).

Los habitantes en su relación histórica con este territorio, han desarrollado conocimientos tradicionales para apropiarse integralmente de sus recursos naturales, de los cuales dependen en diferente grado para su subsistencia. En este sentido, la diversificación productiva que incluye actividades agrícolas, ganaderas y forestales representan la estrategia básica de subsistencia de los hogares en esta región. La agricultura y la ganadería son las principales actividades productivas. La mayoría de 
las localidades practican la agricultura de temporal. Con excepción de comunidades como Limón, Ixtlilco El Chico, Ixtlilco El Grande, Quilamula, Nexpa y una parte de la Tigra que tienen sistema de riego. Los principales cultivos son maíz, calabaza y frijol, los cuales son principalmente para el autoabastecimiento familiar; además de sorgo para la cría de ganado (CONANP 2005). La ganadería puede ser extensiva o de traspatio. En la ganadería extensiva se crían bovinos, equinos, caprino y ovinos. Durante los meses de temporal el ganado pastorea por las superficies de agostadero. En la época de estiaje y posterior a la cosecha de los diferentes cultivos, es alimentado con los esquilmos de los campos agrícolas además de alimento procesado (CONANP 2005). Entre las actividades forestales que realizan los habitantes de la región se encuentra la extracción de leña, recolección de frutos silvestres y plantas medicinales, extracción de copal (Bursera bipinnata, Bursera copallifera) y la cacería de subsistencia (Maldonado 1997, Cruz-León 2008, Cruz-León et al. 2016, Yescas-Albarrán et al. 2016, Abad-Fitz et al. 2020). En las comunidades donde se han construido represas se realiza la pesca, principalmente de mojarra tilapia (Oreochromis spp.) y en el cauce del rio Amacuzac se realiza la pesca de bagre (Ictalurus balsanus) (Domínguez 2015).

La organización social de las comunidades gira en torno a los ejidos. En México, el ejido es un tipo de tenencia de la tierra de propiedad social producto del reparto de latifundios posterior a la revolución mexicana. La superficie ejidal puede estar dividida en parcelas administradas de forma individual por campesinos reconocidos jurídicamente como ejidatarios, tierras de uso común aprovechadas de forma colectiva por miembros de una comunidad y la superficie de los asentamientos humanos (Romero-Navarrete 2015). En la REBIOSH, la mayoría de las comunidades poseen superficie ejidal con excepción de El Zoquital, El Mango y Los Tanques que carecen de dotación oficial.

En las superficies ejidales de varias comunidades se han establecido Unidades de Manejo para la Conservación de la Vida Silvestre (UMA) tales como La Tigra, El Limón, Ixtlilco el Chico, Los Sauces, Pitzotlán, Ixtlilco El Grande, Ajuchitlán, Chimalacatlán, Huautla, Huaxtla, Huixastla, Nexpa, Quilamula, Rancho Viejo, San José de Pala, Santiopan, Xicatlacotla, Pueblo Viejo, Xochipala, Tilzapotla y Chisco (Juárez-Mondragón 2019). La implementación de este instrumento en la política pública mexicana fue parte del Programa de Conservación de la Vida Silvestre y Diversificación Productiva en el Sector Rural 1997-2000. La Ley General de Vida Silvestre establece que las UMA son predios e instalaciones registrados ante la Secretaría del Medio Ambiente y Recursos Naturales, que operan con base en un plan de manejo aprobado y dentro de los cuales se da seguimiento permanente al estado del hábitat, las poblaciones o ejemplares que ahí se distribuyen (DOF 2018). Su objetivo es ser una alternativa para la conservación de la biodiversidad y una oportunidad de diversificación económica para la población rural. Así mismo decreta que sólo a través de este instrumento se permite el aprovechamiento de ejemplares, partes y derivados de vida silvestre (DOF 2018). Las actividades cinegéticas comerciales son una de las principales bajo este esquema (Gallina-Tessaro et al. 2009).

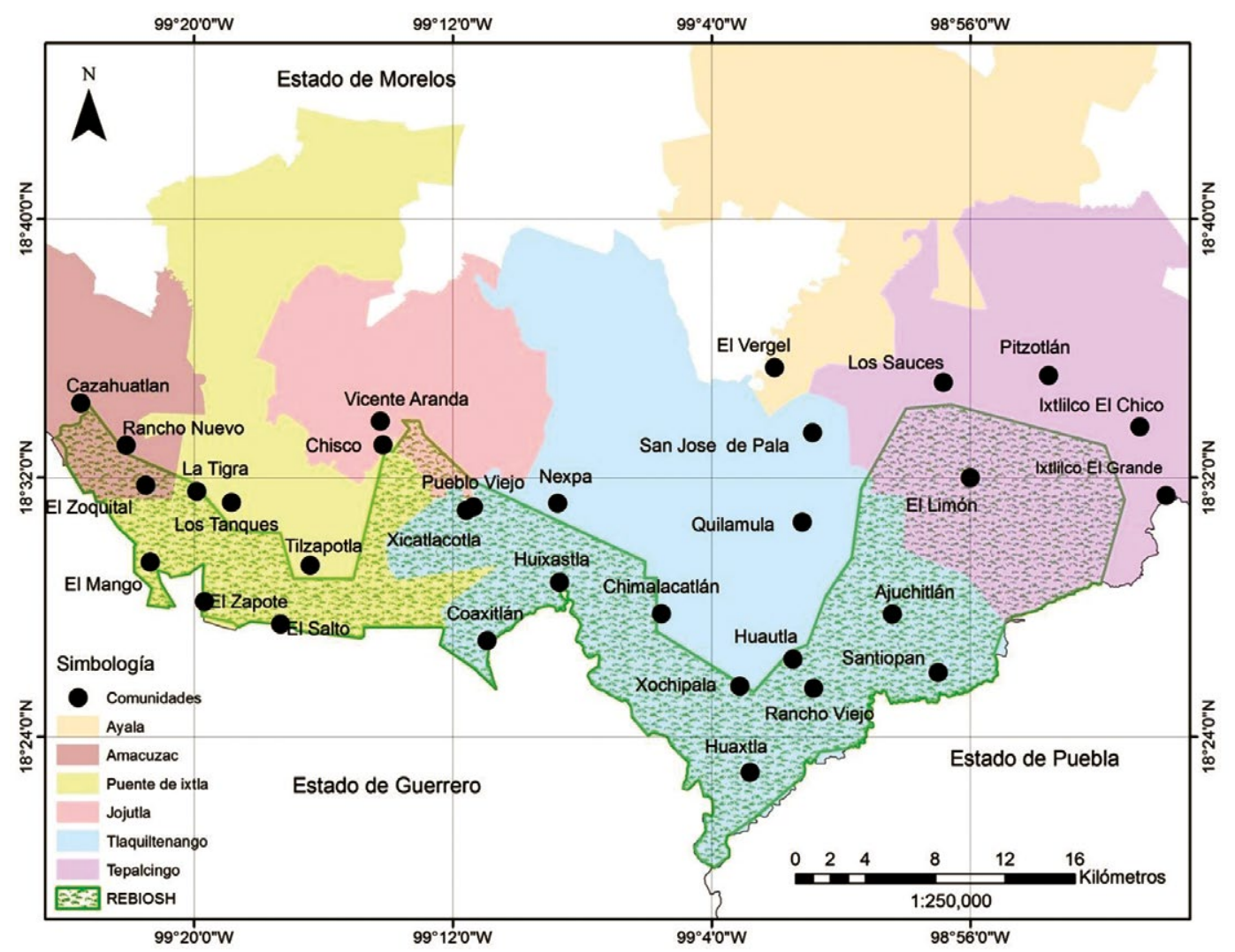

Figura 1. Ubicación de la REBIOSH y comunidades que la integran de acuerdo con CONANP (elaboración propia con datos vectoriales en formato Shapefile obtenidos de CONABIO). 
Método. - En este trabajo se retoma el concepto de apropiación de fauna silvestre para integrar términos que generalmente aparecen en la literatura científica como manejo, uso, aprovechamiento o cacería. La apropiación es la acción por la cual los seres humanos extraen un "fragmento de naturaleza" mediante el trabajo para volverlo un componente social (Toledo 2008). Se realizó una investigación documental sobre estudios de la apropiación de fauna silvestre en comunidades de la Reserva de la Biosfera Sierra de Huautla durante los últimos 20 años (2000-2020). Para este propósito se consultó en buscadores electrónicos como Google académico, Base de datos de la biblioteca virtual del Consorcio Nacional de Recursos de Información Científica y Tecnológica (CONRICyT), bases bibliográficas de la Universidad Autónoma del Estado de Morelos, Universidad Autónoma Chapingo y Universidad Nacional Autónoma de México, en los cuales se buscaron artículos, libros, capítulos de libros, tesis o memorias en extenso de congresos.

Los criterios de inclusión fueron: 1. el estudio fuera en una de las 30 comunidades pertenecientes a los municipios de la declaratoria oficial (CONANP 2005); 2. La apropiación de fauna fuera parte del objetivo del estudio y 3. que proporcionara información sobre las especies con valor de uso. Para evitar el sesgo de información, en el caso de trabajos que se presentaron en congresos o tesis, antes de la publicación de un artículo, se decidió utilizar esta última fuente de información. También se consideró información no publicada generada a partir de técnicas de investigación etnozoológica como entrevistas semiestructuradas, observación etnográfica y registro de la cacería durante siete meses en hogares de una comunidad de la REBIOSH. De las investigaciones recopiladas durante la búsqueda de literatura científica se consideró información como los valores de uso, partes aprovechadas, técnicas e instrumentos de apropiación.

Los valores de uso se describen a partir de la conceptualización de Pérez-Gil et al. (1995), quienes los define como el valor directo y tangible que los usuarios de los recursos naturales obtienen para subsistir. Mientras que el mercadeo o intercambio comercial se define como valor de cambio.

La información recopilada se capturo en una base de datos de Excel. La actualización de los nombres científicos se realizó con la consulta de bases como Avibase - The World Bird Database (https://avibase.bsc-eoc.org/ avibase.jsp?lang=EN), ASM Mammal Diversity Database (https://mammaldiversity.org/), The Reptile Database (http://www.reptile-database.org/), Fishdatabse (https://www.fishbase.in/search.php). Se consultó la NOM-059-SEMARNAT-2010 para identificar las especies clasificadas en alguna categoría de riesgo (DOF 2010).

\section{Resultados y discusión}

Trabajos sobre apropiación de fauna. - Son pocas las publicaciones que se registraron sobre la apropiación de fauna silvestre en Sierra de Huautla (n=11). Las publicaciones documentan la apropiación de fauna en 9 comunidades de la región (Tabla 1). Se distribuyen en seis tesis, cuatro artículos de investigación y un libro. Sin embargo, del total de tesis, tres se publicaron en su respectivo artículo, y una además se publicó en un libro. En comparación con investigaciones de otros recursos como plantas con valor de uso, donde incluso se han realizado estudios regionales a nivel de la Reserva o en la parte alta de la Cuenca del Balsas (Maldonado 1997, Maldonado 2013), la fauna silvestre ha recibido menos atención en el estudio de la relación sociedad-naturaleza en Sierra de Huautla. En este escenario, Naranjo y Cuarón (2010) mencionan que entre las necesidades de investigación en las selvas secas del Pacífico mexicano, como Sierra de Huautla que forma parte de la Cuenca del Balsas, se encuentra la generación de información cualitativa y cuantitativa sobre las características del aprovechamiento de fauna, su impacto en la distribución, abundancia y estructura de las poblaciones faunísticas; así como los efectos de la pérdida y fragmentación del hábitat sobre las especies con mayor frecuencia de uso por los pobladores locales.

Tabla 1. Literatura con información sobre la apropiación de fauna silvestre en Sierra de Huautla, Morelos.

\begin{tabular}{ll}
\hline Comunidades & Trabajos publicados \\
\hline Ajuchitlán & $\begin{array}{l}\text { Juárez et al. 2015, Juárez 2017, Juárez } \\
\text { 2019, Sánchez, 2019. }\end{array}$ \\
\hline Chimalacatlán & $\begin{array}{l}\text { Velarde 2010, Velarde \& Cruz 2015, } \\
\text { Hernández et al. 2018. }\end{array}$ \\
\hline El Limón de Cuachichinola & $\begin{array}{l}\text { Velarde 2010, Velarde \& Cruz 2015, } \\
\text { Bustos et al. 2019. }\end{array}$ \\
\hline El Zoquital & Bello 2015. \\
\hline Los Sauces & $\begin{array}{l}\text { Velarde 2010, Velarde \& Cruz 2015, } \\
\text { Bustos et al. 2019. }\end{array}$ \\
\hline Nexpa & Palacios 2012. \\
\hline Pitzotlán & Valle 2017, García-Flores et al. 2018. \\
\hline San José de Pala & Palacios 2012. \\
\hline Xicatlacotla & Paredes 2013, Domínguez 2015 \\
\hline
\end{tabular}

Una de las probables causas del limitado análisis del aprovechamiento tradicional de fauna en la región se deba a que, como parte del decreto de Reserva de la Biosfera y a la normatividad de la Ley General de Vida Silvestre, se han establecido Unidades de Manejo para la Conservación de la Vida Silvestre (UMA) en modalidad extensiva dentro de las superficies ejidales, las cuales determinan los límites del aprovechamiento de especies animales principalmente de venado cola blanca (Odocoileus virginianus). De hecho, habitantes de la región conceptualizan lo que es una UMA con el "cuidado de los venados" (Juárez-Mondragón et al. 2015). Las UMA no consideran la relevancia sociocultural que tiene la apropiación tradicional de fauna para las comunidades campesinas, limitando el acceso al recurso faunístico a personas que tienen la solvencia económica para la compra de los permisos en un modelo de mercantilización de la vida silvestre (Cruz-Aguilar 2018). A pesar de que se ha documentado que habitantes de la REBIOSH se encuentran a favor de implementar acciones de conservación, también tienen posturas de que algunas de estas restringen su libertad de aprovechar los recursos 
naturales e incluso les parecen excesivas al restringir el acceso a ellos, principalmente el abrir nuevas superficies para la agricultura, la extracción de leña y la práctica de la cacería (Durand 2010, Saldaña-Fernández 2012, Burgos- López-Medellín et al. 2017, Herrera et al. 2019, Sánchez 2019). Este aspecto es relevante en el contexto de que su preocupación conservacionista está estrechamente relacionada con las condiciones que les permitan reproducir su vida material, en función de la apropiación de su entorno para la satisfacción de sus necesidades y asegurar la subsistencia (Durand 2010, López-Medellín et al. 2017, Trujillo-Santisteban \& López-Medellín 2018, Burgos-Herrera et al. 2019).

Analizar el éxito y eficacia de las políticas públicas de conservación como la UMA puede resultar ambiguo. En el caso particular del estado de Morelos, la evaluación de esta estrategia se limita por la falta de información ante la carencia de mecanismos de seguimiento y supervisión (González-Rebeles et al. 2020). En la REBIOSH, como parte de las estrategias de conservación, probablemente han obtenido resultados en el manejo del hábitat, porque en los últimos años se registra un incremento en la superficie forestal de selva baja caducifolia (Sorani et al. 2020) y los habitantes perciben aumento en las poblaciones animales y monte (Durand 2010, López-Medellín et al. 2017, García-Flores et al. 2018, Sánchez 2019). Sin embargo, uno de sus objetivos principales como lo es ser una alternativa para el desarrollo de las comunidades ha sido limitado (Pérez-Soto 2016, Cruz-Aguilar et al. 2018). Incluso en algunas localidades de la región se manifiesta desmotivación en este modelo mercantil por el poco turismo cinegético para la venta de permisos de cacería deportiva (Juárez-Mondragón et al. 2015), lo cual se integra a problemáticas como datos pocos confiables de las poblaciones de venado, falta de individuos que puedan servir como trofeos de caza rentables, pocos subsidios y apoyos gubernamentales, conflictos comunitarios por la distribución de los ingresos, falta de infraestructura y difusión (Pérez-Soto et al. 2016, Pérez-Soto 2016). En el contexto económico, Gallina-Tessaro et al. (2009) mencionan que las condiciones de las UMA del norte del país como grandes superficies de terreno, esquemas de ganadería diversificada, experiencia en actividades cinegéticas y la cercanía a la frontera con Estados Unidos donde provienen cazadores con poder adquisitivo, les ha permitido tener una alta rentabilidad económica, situación contraria a las UMA del centro y sureste de México.

La apropiación de fauna silvestre como parte de las estrategias de subsistencia campesina ha representado una actividad incomprendida y subestimada (Santos-Fita 2018). Se concibe como ilegal cuando se realiza fuera del esquema de UMA, como especifica el plan de manejo de la REBIOSH (CONANP 2005). Este contexto de ilegalidad puede dificultar las investigaciones sobre la relación sociedad-fauna en la región, porque las personas prefieren abstenerse de participar en estudios o compartir información referentes a la apropiación de fauna por miedo a represalias de autoridades ambientales (Tejeda-Cruz et al. 2014), tal como se ha registrado en algunas localidades de Sierra de Huautla, donde en primera instancia se omite la práctica de la cacería como parte de las estrategias de subsistencia (Sánchez 2019). Esta condición limita la generación de información sobre las especies de fauna aprovechadas o de investigaciones más puntuales como la estimación de la extracción, a pesar de que el plan de manejo especifica que se deben impulsar las investigaciones etnobiológicas como apoyo para la adecuada gestión de los recursos naturales (CONANP 2005).

En este escenario de dificultades metodológicas que podrían impedir el estudio de la apropiación de fauna, se sugiere un trabajo de campo extenso y estancias en las localidades que permitan generar una relación de confianza y conexión con los habitantes. Así mismo dejar claro los objetivos de las investigaciones, el manejo de la información y la confidencialidad a los informantes. Se deben considerar la implementación de múltiples técnicas para la obtención de información como entrevistas a profundidad, observación participante, elaboración de mapas participativos y recorridos etnobiológicos para construir conocimientos genuinos de la realidad de los colaboradores y disminuir el riesgo de encontrar conclusiones equivocadas al recoger información solo por encuestas o cuestionarios (Medrano 2012).

Un aspecto general que en los últimos años ha sido objeto de discusión en diferentes ámbitos de investigación social es el enfoque "extractivista". En el caso de la REBIOSH, por ejemplo, Sánchez (2019) reporta que habitantes expresan que llegan estudiantes e investigadores de distintas universidades y solo les "sacan información y luego nada", ya que las autoridades no hacen nada al respecto de las problemáticas, lo que genera un ambiente de molestia o mencionan que el trato que reciben de los estudiantes e investigadores es solo como cargadores. En dicho contexto, Sánchez (2019) propone que las universidades deben desarrollar programas de educación y sensibilización para los trabajos en campo y la relación con los habitantes de las localidades. Sin embargo, esos aspectos deben ser inherentes desde el principio en que se diseña una investigación etnobiológica o de alguna área afín, porque el trabajo etnobiológico desarrollado por investigadores latinoamericanos debe tener generalmente un compromiso social diferente al de etnobiólogos de otras partes del mundo (Cano et al. 2014). El investigador no solo va a conocer la realidad de su área de estudio, también convive y es partícipe de lo que investiga, no debe ser un sujeto ajeno, frio o indiferente. El comienzo verdadero de la investigación es cuando el investigador es aceptado en el círculo social de los colaboradores, para lo cual la directriz en la relación debe ser una entre seres humanos y no de un investigador con un objeto de estudio (Alarcón-Chaires \& Toledo 2003). Así mismo, es importante que se establezcan estrategias de devolución de resultados a las comunidades e incluso se pueden establecer compromisos realistas de cooperación en ámbitos no necesariamente relacionados con el trabajo de investigación.

Formas de apropiación. - La apropiación de fauna silvestre se realiza por medio de la práctica de la cacería, la recolección y la pesca. Las armas de fuego como 
la escopeta es uno de los principales instrumentos, sin embargo, también se emplean perros, machetes, resorteras, nudos corredizos o se capturan presas manualmente (Velarde \& Cruz 2015, Bello 2015, Valle 2017). La pesca de bagre (Ictalurus balsanus) es mediante la utilización de utensilios artesanales como la tarraya y anzuelo. La práctica de la caza puede ser una actividad oportunista cuando los campesinos se encuentran animales mientras realizan actividades agrícolas, ganaderas o forestales (Bello 2015, Valle 2017). Pero también es una actividad organizada, como lo demuestran técnicas de apropiación como las arreadas, lampareada y espiada. Estas modalidades tradicionales de caza registradas en comunidades de la reserva, también se han descrito en localidades del sureste de México (Ramírez-Barajas \& Calme 2015). Las técnicas de apropiación se encuentran relacionadas con las características biológicas y de comportamiento de las especies animales (Santos-Fita et al. 2012). En este contexto, en comunidades de la REBIOSH se ha documentado que los cazadores poseen conocimientos sobre aspectos de la fauna que se apropian como hábitos alimentarios, lugares de refugio y alimentación, dimorfismo sexual, rastros que dejan, patrón de actividad, distribución territorial, época de reproducción, comportamiento social y abundancia (Velarde \& Cruz 2015, Valle 2017). A continuación, se describen estas técnicas de apropiación:

- Arreadas: es una práctica colectiva para la captura del venado cola blanca. Los integrantes que participan pueden variar entre 6 a 30 personas, las cuales se dividen en dos grupos, los tiradores y los arreadores. Son dirigidas por cazadores con mayor experiencia. Se colocan como tiradores a aquellas personas con mayor experiencia en el manejo de la escopeta para que sus disparos acierten a la presa, en los "puestos", sitios con vista amplia sobre el área, que permite visualizar los individuos de venado, diferenciar entre machoshembra y escoger aquellos con mejores características como una cornamenta con mayor número de puntas. Los arreadores entran en el área en línea recta, haciendo ruido para que la presa se dirija hacia los puestos de los tiradores. La carne de las presas obtenidas se reparte de forma equitativa o se consume grupalmente. Además de obtener un complemento alimentario, esta práctica se concibe como un esparcimiento y convivencia comunitaria entre los habitantes locales y amistades de otras comunidades, incluyendo cazadores, mujeres y niños (Velarde \& Cruz 2015, Valle 2017, Juárez 2019).

- Espiada: es una modalidad de cacería individual tanto diurna como nocturna, que consiste en que el cazador identifica un lugar donde una presa se alimenta o bebe agua. En el caso del venado cola blanca se ubican lugares con cazahuate (Ipomea spp.), de cuya flor se alimenta. Se coloca un espiadero sobre un árbol cercano en el cual se espera a que la presa llegue a comer. Las huilotas se capturan con esta técnica cuando el cazador espera en ojos de agua o presas cuando llegan a beber agua. Una variante es la espiada colectiva, donde cazadores simultáneamente se colocan diferentes espiaderos en diferentes sitios, repartiendo la presa obtenida (Bello 2015, Velarde \& Cruz 2015, Valle 2017).

- Lampareada: modalidad nocturna que puede ser individual o grupal en pequeños grupos de hasta cuatro personas. Para la cacería del venado, se realiza en luna llena para tener visibilidad en el monte. Cuando se encuentra la presa, se deslumbra con la luz de una lámpara para cegarla, desorientarla y dispararle. De acuerdo con el conocimiento tradicional, los machos tendrán un reflejo rojo en sus ojos, mientras que las hembras lo tienen azul. En el caso de huilotas (Zenaida spp.) o tórtolas (Columbina spp.), con la lámpara se buscan individuos dormidos en árboles o arbustos, disparándoles con la resortera cuando se localizan (Velarde \& Cruz 2015, Valle 2017).

- Campeada: son caminatas que se realizan para la búsqueda de animales cuando paralelamente se practican actividades agrícolas, pecuarias o forestales (Bello 2015).

- Acorralamiento: una de las formas para capturar a Ctenosaura pectinata es por medio del acorralamiento, para lo cual se le rodea en árboles o tecorrales.

- Recolección manual: se emplea para la apropiación de insectos comestibles.

Riqueza de fauna con valores de uso. - Se registran 46 especies con valor de uso (Tabla 2) (Fig. 2). El Plan de Manejo de la REBIOSH (CONANP 2005) menciona que se consideran a 17 mamíferos, 54 aves, cuatro reptiles, tres anfibios, cinco peces y cuatro insectos con valores de uso. Sin embargo, no presenta ningún listado, no especifica alguna metodología que describa cómo se obtuvo dicha información, ni cita trabajos de investigación realizados como en la descripción de la flora con valores de uso. La apropiación de fauna silvestre provee a los habitantes de Sierra de Huautla de bienes con valores de uso alimentario, medicinal, ornamental, de amuleto, conseguir animales de compañía, materia prima para la elaboración de herramientas y obtener recursos económicos (Fig. 3). El mayor porcentaje de la diversidad de especies apropiadas tiene valor de uso alimentario (63.8\%) y medicinal (38\%). En América Latina, el consumo de fauna silvestre y su uso en la medicina tradicional son prácticas comunes, por ser una alternativa económica, accesible y culturalmente aceptable para la alimentación y tratamiento de enfermedades de la población campesina (Alves \& Alves 2011). La riqueza faunística muestra el aprovechamiento diversificado de la fauna, incluso se registra un mayor número de especies aprovechadas en comparación con habitantes rurales de la Reserva Natural Sierra Nanchititla $(n=33)$, en un contexto similar donde se distribuye selva seca propia de la Cuenca del Balsas y es un estudio regional que considera más de una comunidad (Monroy-Vilchis et al. 2008). 


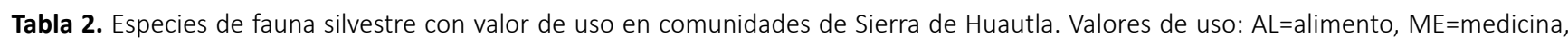
$\mathrm{OR}=$ ornamental, $\mathrm{AM}=$ amuleto, $\mathrm{HE}=$ herramienta, $\mathrm{MA}=$ mascota, $\mathrm{ED}=$ especie dañina, $\mathrm{VN}=$ venta. Partes utilizadas: $\mathrm{CAR}=\mathrm{carne}, \mathrm{VIS}=\mathrm{vísceras}$, $\mathrm{HUE}=$ huesos, $\mathrm{SAN}=$ sangre, $\mathrm{GR}=$ grasa, $\mathrm{AS}=$ astas, $\mathrm{BEZ}=$ bezoar, $\mathrm{PA}=$ patas, $\mathrm{PI}=$ piel, $\mathrm{CAB}=$ cabeza, $\mathrm{ANC}=$ animal completo, $\mathrm{COL}=\mathrm{col} \mathrm{millos}$, $\mathrm{HUE}=$ huevos, $\mathrm{ORI}=$ orina, $\mathrm{CO}=$ cola, $\mathrm{CAP}=$ caparazón, HIE=hiel. *Categoría de riesgo NOM-059-SEMARNAT-2010: Pr= protección especial, A=amenazada.

\begin{tabular}{|c|c|c|c|c|}
\hline Orden/Familia & Nombre Científico & Nombre Común & Valores De Uso & Partes Utilizadas \\
\hline \multicolumn{5}{|l|}{ MAMIFEROS } \\
\hline \multicolumn{5}{|l|}{ Artiodactyla } \\
\hline \multirow{8}{*}{ Cervidae } & \multirow{8}{*}{ Odocoileus virginianus Zimmermann,1780 } & \multirow{8}{*}{ Venado/Cuernicabra/Ciervo } & $\mathrm{AL}$ & CAR, VIS, HUE \\
\hline & & & $\mathrm{ME}$ & SAN, GR, AS, BEZ \\
\hline & & & OR & AS, PA, PI, CAB \\
\hline & & & AM & BEZ \\
\hline & & & $\mathrm{HE}$ & AS, PA \\
\hline & & & MA & ANC \\
\hline & & & ED & - \\
\hline & & & VN & $\mathrm{PI}, \mathrm{CAB}$ \\
\hline \multirow{3}{*}{ Tayassuidae } & \multirow{3}{*}{ Pecari tajacu Linnaeus, 1758} & \multirow{3}{*}{ Jabalí/Marrano de monte } & $\mathrm{AL}$ & CAR \\
\hline & & & ED & - \\
\hline & & & AM & $\mathrm{COL}$ \\
\hline \multicolumn{5}{|l|}{ Carnívora } \\
\hline \multirow{11}{*}{ Procyonidae } & \multirow{7}{*}{ Nasua narica Linnaeus, 1766} & \multirow{7}{*}{ Tejón } & $\mathrm{AL}$ & CAR, HUE \\
\hline & & & ME & GR, ANC \\
\hline & & & OR & $\mathrm{PI}$ \\
\hline & & & AM & PA \\
\hline & & & MA & ANC \\
\hline & & & ED & - \\
\hline & & & VN & ANC \\
\hline & \multirow{3}{*}{ Procyon lotor Linnaeus, 1758} & & $\mathrm{AL}$ & CAR \\
\hline & & Mapache/ Kailama & AM & PA \\
\hline & & & ED & - \\
\hline & Bassariscus astutus Lichtenstein, 1830 & Cacomixtle & ED & - \\
\hline & & & $\mathrm{AL}$ & CAR \\
\hline & Menhitic macrourg lichtenctoin 1822 & 7orrillo ravado & ME & CAR \\
\hline & IVIepnins macroura Licntensteln los & Lorrillo rayado & ED & - \\
\hline & & & VN & CAR \\
\hline Mephitidae & Spilogale gracilis Merriam, 1890 & Zorrillo pinto & $\mathrm{ME}$ & CAR, GRA, ORI \\
\hline & & & $\mathrm{AL}$ & CAR \\
\hline & & & $\mathrm{ME}$ & CAR \\
\hline & Conepatus leuconotus Lichtenstein, 1832 & Zorrillo cadeno & ED & - \\
\hline & & & VN & CAR \\
\hline & & & ME & - \\
\hline & Urocyon cinereoaraenteus Schreher 1775 & 7orra & ED & - \\
\hline & Urocyon cmereoargenteus scrirever, 1/7 & Zorra & $\mathrm{AL}$ & CAR \\
\hline Canidae & & & VN & CAR \\
\hline camiae & & & ED & - \\
\hline & Caniclatranc Sav 1822 & Covoto & AM & $\mathrm{COL}$ \\
\hline & Canis Iatrans say, $18<3$ & coyote & ME & $\mathrm{CAR}, \mathrm{GR}, \mathrm{COL}$ \\
\hline & & & OR & $\mathrm{COL}$ \\
\hline Lagomorpha & & & & \\
\hline & Sylvilagus cuniculariuc Waterhouse 1848 & Conein & $\mathrm{AL}$ & CAR \\
\hline Leporidae & syıiliagus cunicuiarius vaternouse, 1848 & conejo & MA & ANC \\
\hline & Lepus callotis Wagler, 1830 & Liebre & $\mathrm{AL}$ & CAR \\
\hline Didelphimorph & & & & \\
\hline & & & $\mathrm{AL}$ & CAR \\
\hline Didelnhidae & Didelnhis virainiana Kerr 1792 & Tlacuache & AM & PA \\
\hline & & Tlacuache & $\mathrm{ME}$ & $\mathrm{CO}$ \\
\hline & & & ED & - \\
\hline Cingulata & & & & \\
\hline & & & $\mathrm{AL}$ & CAR \\
\hline Dasypodidae & Dasypus novemcinctus Linnaeus, 1758 & Armadillo & $\mathrm{ME}$ & GR, CAP, CAR \\
\hline & & & OR & CAP \\
\hline Rodentia & & & & \\
\hline Sciuridae & Sciurus aureogaster PF. Cuvier, 1829 & Ardilla & $\mathrm{AL}$ & CAR \\
\hline AVES & & & & \\
\hline Columbiformes & & & & \\
\hline & Zenaida asiática Linnaeus, 1758 & $\begin{array}{l}\text { Paloma de alas blancas/ cocole- } \\
\text { ra/ huilota }\end{array}$ & $\mathrm{AL}$ & CAR \\
\hline & Zenaida macroura Linnaeus. 1758 & 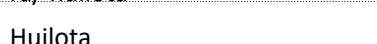 & $\mathrm{AL}$ & CAR \\
\hline Columbidae & Zenaiaa macroura Linnaeus, 1/s & Huliota & VN & CAR \\
\hline & Columbing incalecson 1847 & Tórtola & $\mathrm{AL}$ & CAR \\
\hline & Columbina inca Lesson, $184 /$ & Iortola & MA & ANC \\
\hline & Columbina passerina Linnaeus, 1758 & Cocoquita/tortola & $\mathrm{AL}$ & CAR \\
\hline & Leptotila verreauxi Bonaparte, 1855 & Paloma/ Barranquera/ Pedorra & $\mathrm{AL}$ & CAR \\
\hline
\end{tabular}




\begin{tabular}{|c|c|c|c|c|}
\hline Orden/Familia & Nombre Científico & Nombre Común & Valores De Uso & Partes Utilizadas \\
\hline \multicolumn{5}{|l|}{ Galliformes } \\
\hline \multirow{2}{*}{ Odontophoridae } & \multirow{2}{*}{ Philortyx fasciatus Gould, 1844} & \multirow{2}{*}{ Codorniz/ Churrunda } & $A L$ & CAR \\
\hline & & & MA & ANC \\
\hline \multirow{2}{*}{ Cracidae } & \multirow{2}{*}{ Ortalis poliocephala Wagler, 1830} & \multirow{2}{*}{ Chachalaca/ Paita/ Cuira } & $A L$ & CAR \\
\hline & & & MA & ANC \\
\hline \multicolumn{5}{|l|}{ Cuculiformes } \\
\hline Cuculidae & Geococcyx velox Wagner, 1836 & Correcaminos & $\mathrm{ME}$ & CAR \\
\hline \multicolumn{5}{|l|}{ Piciformes } \\
\hline Picidae & Melanerpes chrysogenys Vigors, 1839 & Carpintero/ La chica & ME & ANC \\
\hline \multicolumn{5}{|l|}{ Passeriformes } \\
\hline Turdidae & Turdus rufopalliatus Lafresnaye, 1840 & Primavera & $\mathrm{AL}$ & CAR \\
\hline \multirow{2}{*}{ Passerellidae } & Peucaea ruficauda Bonaparte, 1853 & Comadrita & $\mathrm{AL}$ & CAR \\
\hline & Peucaea humeralis Cabanis, 1851 & Campanero & $\mathrm{AL}$ & CAR \\
\hline \multicolumn{5}{|l|}{ Anseriformes } \\
\hline \multirow{2}{*}{ Anatidae } & \multirow{2}{*}{ Anas spp. } & \multirow{2}{*}{ Pato } & $\mathrm{AL}$ & CAR \\
\hline & & & MA & ANC \\
\hline \multicolumn{5}{|l|}{ Falconiformes } \\
\hline Falconidae & Caracara plancus Miller, JF, 1777 & Quebrantahuesos & ED & - \\
\hline \multicolumn{5}{|l|}{ Accipitriformes } \\
\hline & Buteo nitidus Latham, 1790 & Gavilán pollero & ED & - \\
\hline Accipitridae & Buteo jamaicensis Gmelin, JF, 1788 & Gavilán colorado & ED & - \\
\hline & Parabuteo unicinctus Temminck, 1824 & Aguililla (Pr)* & ED & - \\
\hline Cathartiformes & & & & \\
\hline & Coragyps atratus Bechstein, 1793 & Zopilote & $\mathrm{ME}$ & CAR \\
\hline Cathartidae & Cathartes aura Linnaeus, 1758 & Zopilote, aura & $\mathrm{ME}$ & CAR \\
\hline Pelecaniformes & & & & \\
\hline Ardeidae & Ardeidae sp. & Garza & $\mathrm{AL}$ & CAR \\
\hline REPTILES & & & & \\
\hline Squamata & & & & \\
\hline & & & $\mathrm{AL}$ & CAR \\
\hline Iquanidae & Ctenocaurg nestinata Wliegmann 1824 & |ศнара $(\Delta) *$ & ME & CAR, SAN \\
\hline Iguanidae & Ctenosaura pectnata wiegmann, 1834 & $\operatorname{Iguana}(A)^{*}$ & MA & ANC \\
\hline & & & VN & CAR, ANC \\
\hline & & & $A L$ & CAR \\
\hline & & & $\mathrm{ME}$ & CAR, PI, HIE \\
\hline Klineridan & Crotalus culminatuc Klauher 1952 & Víhora de cascabol & OR & $\mathrm{PI}-2$ \\
\hline VIperıaae & crotaius culminatus Klauder, 1952 & Vidora de cascabel & MA & ANC \\
\hline & & & VN & ANC \\
\hline & & & ED & - \\
\hline Elapidae & Micrurus laticollaris Peters, 1870 & Coralillo & ED & - \\
\hline Boidae & Bog constrictor Linnaeus 1758 & Mazacuate & ED & - \\
\hline & & & OR & $\mathrm{PI}$ \\
\hline Testudines & & & & \\
\hline & & & $\mathrm{AL}$ & CAR \\
\hline Kinosternidae & Kinosternon integrum PR Le Conte,1925 & Tortuga $(\mathrm{Pr})^{*}$ & ED & - \\
\hline & & & $\mathrm{ME}$ & ANC, SAN \\
\hline ANFIBIOS & & & & \\
\hline Anura & & & & \\
\hline Rufonidan & Rhinella marina Linnaeus, 1758 & Sapo & $\mathrm{ME}$ & ANC \\
\hline Butonidae & Anaxyrus punctatus Baird \& Girard, 1852 & Sapo & MA & ANC \\
\hline Hylidae & Agalychnis dacnicolor Cope, 1864 & Rana & ME & ANC \\
\hline INSECTOS & & & & \\
\hline Orthoptera & & & & \\
\hline Pyrgomorphidae & Sphenarium sp. & Chapulín/Contapa & $\mathrm{AL}$ & ANC \\
\hline Hemiptera & & & & \\
\hline Pentatomidae & Euschistus sulcacitus Rolston, 1974 & Jumil/ Chumil & $\mathrm{AL}$ & ANC \\
\hline PECES & & & & \\
\hline Siluriformes & & & & \\
\hline Ictaluridae & Ictalurus balsanus (Jordan \& Snyder, 1899) & Bagre & $\mathrm{AL}, \mathrm{VN}$ & CAR \\
\hline
\end{tabular}

Las especies pertenecen a 31 familias faunísticas. El orden Carnívora $(\mathrm{n}=8)$, Columbiforme $(\mathrm{n}=5)$ y Squamata $(n=4)$ son los que mayor número de especies contabilizan. La clase faunística con mayor número de especies apropiadas son las aves $(42.5 \%)$, seguidas de los mamíferos (34\%), reptiles $(10.6 \%)$, anfibios (6.4\%), insectos $(4.3 \%)$ y peces $(2.1 \%)$. En diferentes lugares del Neotrópico, las aves y mamíferos son los grupos que mayormente se aprovechan (Herrera-Flores et al. 2019). Esto podría deberse al mayor aporte de productos, como el caso de los mamíferos, donde el $73 \%$ de los registrados en esta revisión son de uso múltiple, destacando 0 . virginianus con seis valores de uso y partes comercializadas. En comunidades de la REBIOSH el venado cola blanca es la especie preferida y apreciada en la práctica de la cacería e incluso la normatividad institucional y comunitaria se encuentran enfocada a su alrededor (Velarde \& Cruz 2015, Juárez-Mondragón et al. 2015, García-Flores 
et al. 2018). Este cérvido es culturalmente importante en comunidades rurales de México, principalmente por su aporte de proteína y el gusto por su carne es muy arraigado en diferentes regiones del país (López et al. 2005). De hecho, en la comunidad de Pitzotlán es considerado como una de las carnes más sabrosas (Valle 2017). Ade- más, es relevante por su aprovechamiento integral donde se obtiene productos medicinales, materias primas para herramientas y ornamentos (Estrada-Portillo et al. 2018), similar a lo registrado en este trabajo donde se reporta el aprovechamiento de astas, grasa, sangre, piel, cabezas o patas para diversos fines similares.
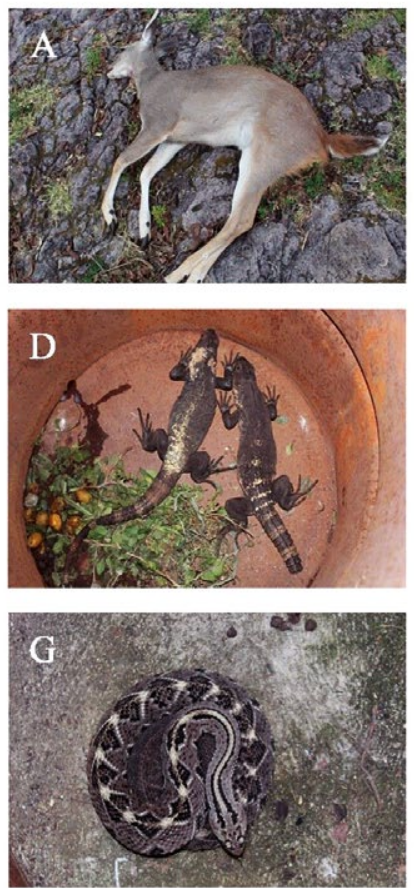
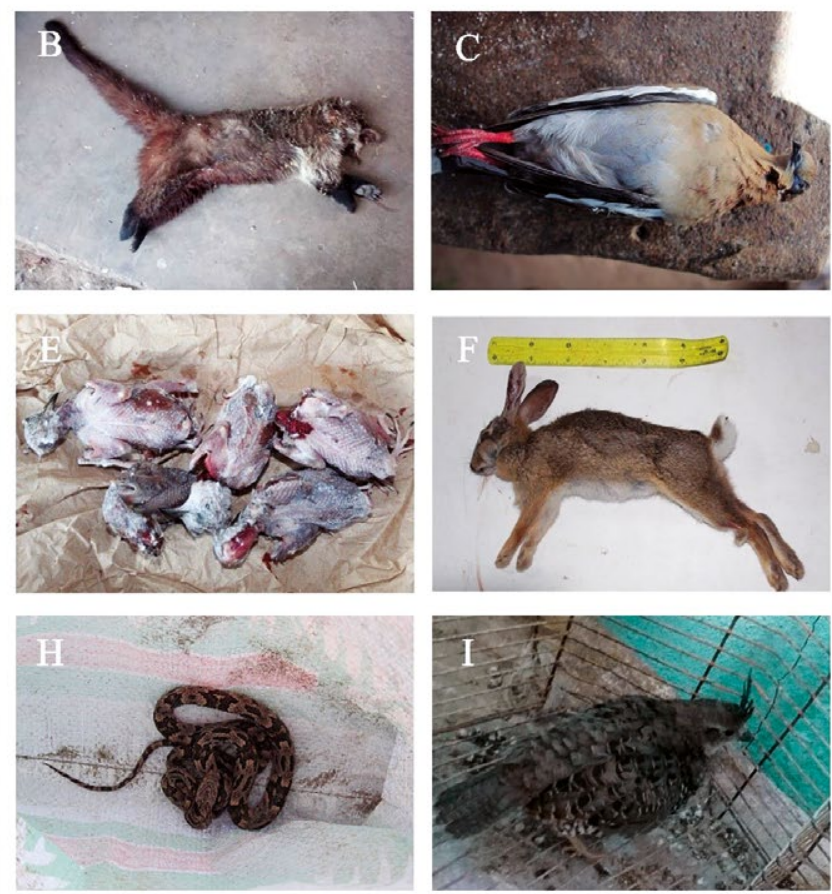

Figura 2. Ejemplos de fauna silvestre apropiada en Sierra de Huautla: A. venado cola blanca (Odocoileus virginianus), B. tejón (Nasua narica), C. huilota o paloma de alas blancas (Zenaida asiática), D. iguana (Ctenosaura pectinata), E. tórtolas (Columbina inca), F. conejo de campo (Sylvilagus cunicularius), G. víbora de cascabel (Crotalus culminatus), H. mazacuate (Boa constrictor), I. codorniz (Philortyx fasciatus). Fotos obtenidas en trabajo de campo.
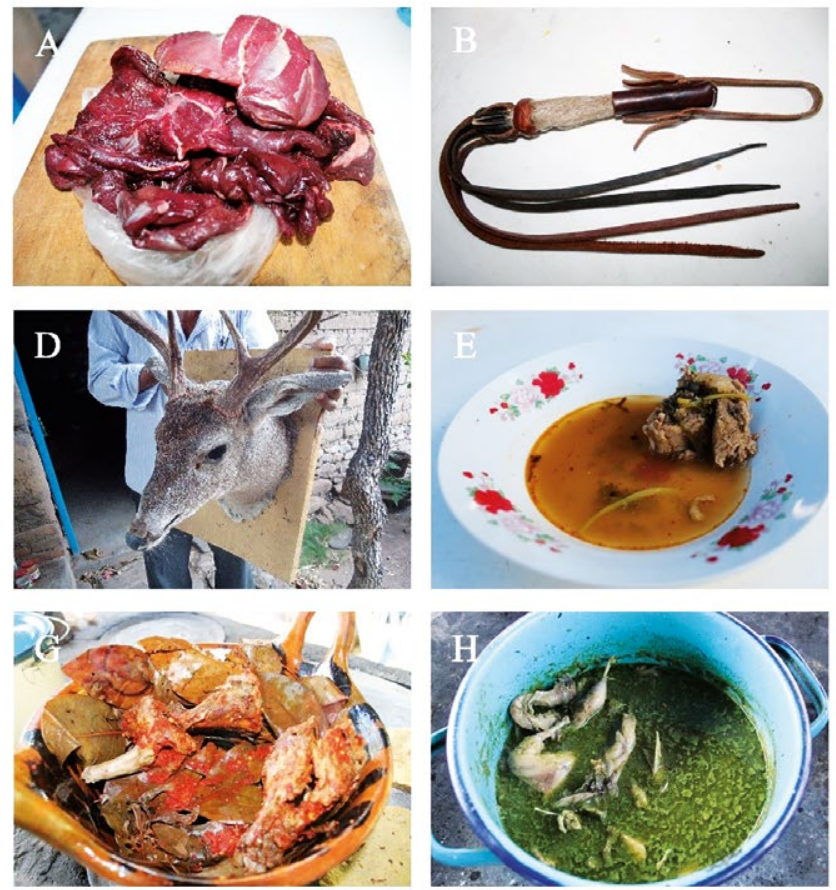
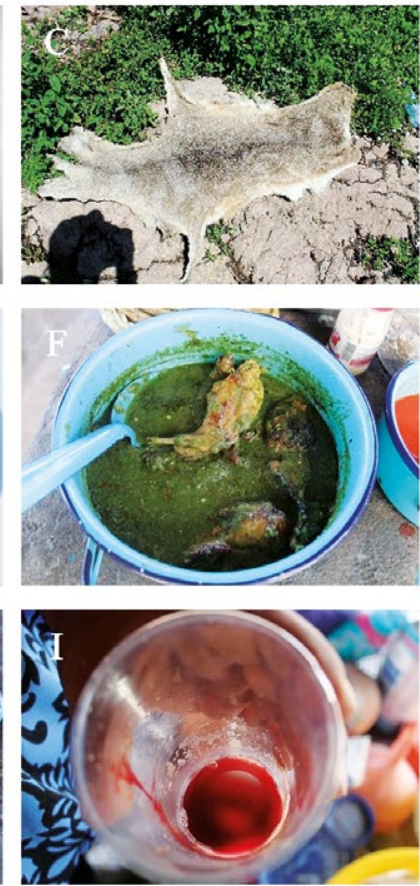

Figura 3. Ejemplos de bienes proporcionados por la fauna silvestre en Sierra de Huautla: A. carne de venado, B. fuete para caballo con pata de venado, C. piel de venado, D. trofeo de cacería, E. guaxmole con carne de venado, F. carne de conejo en salsa verde, G. carne de tejón cocida en hojas de aguacate, H. carne de codorniz en salsa verde, I. sangre de iguana para uso medicinal. Fotos obtenidas en trabajo de campo. 
Alimento. - En la región, la fauna provee un aporte de proteína animal como parte de la cultura alimentaria de los habitantes. Con la carne, vísceras y huevos se elaboran platillos de la gastronomía regional en diferentes formas de preparación como caldos, asado, salsas, barbacoa o tlaquemal, guaxmole, bistecs, carne seca, carne frita, asadura y mole (García-Flores et al. 2018). El uso alimentario es el principal motivo del aprovechamiento de la fauna silvestre en diversas partes de México y en general en Latinoamérica, al representar una alternativa de proteína animal para el complemento de la alimentación de la población rural (Guerra et al. 2010). En regiones del trópico húmedo mexicano se ha estimado que, en la dieta de pobladores rurales, la carne proveniente de la fauna silvestre aporta hasta el $50 \%$ de la proteína (Tejeda-Cruz et al. 2014). En el trópico seco y en el caso particular de Sierra de Huautla este tipo de información es casi nula, con excepción de algunas especies comestibles como la iguana (Ctenosaura pectinata) y el bagre (Ictalurus balsanus). Sin embargo, más que cuantificar el aporte proteico a la dieta, se ha estimado la extracción de individuos. En el Limón de Cuauchichinola y Los Sauces se ha estimado la extracción para alimento de hasta 110 individuos de iguana en un año, particularmente hembras con huevos en el oviducto, cuyo consumo forman parte de la cultura alimentaria regional (BustosZagal et al. 2019). En la comunidad Xicatlacotla la pesca tradicional de bagre en el rio Amacuzac, especie endémica de la Cuenca del Balsas, tiene gran importancia socioeconómica por su aporte para la alimentación y obtención de ingresos económicos por su mercadeo local (Domínguez 2015, Paredes-Lira 2013). Evaluaciones cuantitativas de la pesca de esta especie en la comunidad muestran que anualmente se pueden obtener hasta $1494.93 \mathrm{~kg}$ de peso vivo con ganancia de $\$ 74,746.5$ pesos mexicanos (Domínguez 2015)

Medicina tradicional. - El 38\% de las especies registradas se utilizan para el tratamiento de padecimientos en la medicina tradicional (Tabla 3) como la vista cansada, el acné, hemorragias, anemia, bronquitis, asma, tos, reumas, dolores de cabeza, picaduras de alacrán, hipertensión, alergias, diabetes, cáncer, epilepsia, y enfermedades de filiación cultural como mal de ojo, frio, calor, empacho y aire. A pesar de que en la práctica de la medicina tradicional mexicana el uso de plantas predomina, la utilización de animales con fines terapéutico es importante en comunidades del país (CONABIO 2020), como los diversos usos medicinales en Sierra de Huautla. Por ejemplo, la carne de C. pectinata se consume para evitar la anemia y mejorar la vista, su sangre se toma para reponer a mujeres embarazadas. El consumo de carne de zorrillos es para el tratamiento de acné y alergias cutáneas. En tanto la grasa de $O$. virginianus se emplea para afecciones respiratorias como asma y dolor en articulaciones por frialdad. La diversidad de aplicaciones medicinales del venado cola blanca contrasta con otras regiones de Morelos como el Corredor Biológico Chichinautzin, la Reserva Estatal Sierra Montenegro y el Parque Nacional El Tepozteco, donde no se ha registrado su uso terapéutico (García-Flores 2008, Monroy et al. 2011, García-Flores et al. 2014).

Tabla 3. Fauna silvestre con valor de uso medicinal registrada en comunidades de Sierra de Huautla.

\begin{tabular}{|c|c|c|c|}
\hline Especie & Enfermedad & Parte aprovechada & Modo de empleo \\
\hline \multirow{12}{*}{ Odocoileus virginianus } & Hipertensión & Sangre & $\begin{array}{l}\text { La sangre se ingiere fresca o seca diluida en jugo de } \\
\text { frutas }\end{array}$ \\
\hline & Bronquitis y asma & Grasa & $\begin{array}{l}\text { La grasa es usada como ungüento que es aplicada en } \\
\text { el pecho del enfermo }\end{array}$ \\
\hline & Epilepsia & Sangre & $\begin{array}{l}\text { Se ingiere la sangre del animal, preferentemente fresca } \\
\text { poco después de capturar al animal }\end{array}$ \\
\hline & Aire en infantes & Bezoar gastrointestinal & $\begin{array}{l}\text { Se realiza una limpia al infante con el bezoar por todo } \\
\text { el todo cuerpo }\end{array}$ \\
\hline & Dolor de cabeza & Astas & $\begin{array}{l}\text { Se realiza un masaje sobre la cabeza con un pedazo } \\
\text { de asta }\end{array}$ \\
\hline & Dolor de espolón calcáreo & Astas & $\begin{array}{l}\text { Se realiza un masaje sobre el área del espolón con un } \\
\text { pedazo de asta }\end{array}$ \\
\hline & Frio en articulaciones & Grasa & La grasa se unta sobre las articulaciones afectadas \\
\hline & Mala vista & Carne, sangre & Se consume su carne \\
\hline & Anemia & Sangre & Se consume su sangre \\
\hline & Dolores musculares & Grasa & Se aplica en zonas afectadas \\
\hline & Tos ahogona & Grasa & Sin especificar modo de uso \\
\hline & Frio & Grasa & Sin especificar modo de uso \\
\hline \multirow{4}{*}{ Mephitis macroura } & Alergias cutáneas & Carne & $\begin{array}{l}\text { Se consume la carne en caldo o también el caldo puede } \\
\text { usarse para bañarse }\end{array}$ \\
\hline & Mal de ojo & Carne & Se consume la carne frita o en caldo \\
\hline & Acné & Carne & Se consume su carne frita o en caldo \\
\hline & Asma & Carne & Se consume la carne en caldo \\
\hline \multirow{6}{*}{ Conepatus leuconotus } & Alergias cutáneas & Carne & $\begin{array}{l}\text { Se consume la carne en caldo o también el caldo puede } \\
\text { usarse para bañarse }\end{array}$ \\
\hline & Mal de ojo & Carne & Se consume la carne frita o en caldo \\
\hline & Acné & Carne & Se consume su carne frita o en caldo \\
\hline & Asma & Carne & Se consume la carne en caldo \\
\hline & Asma y bronquitis & Olor & Sin especificar modo de uso \\
\hline & Tos ahogona & Carne & Sin especificar modo de uso \\
\hline
\end{tabular}




\begin{tabular}{|c|c|c|c|}
\hline Especie & Enfermedad & Parte aprovechada & Modo de empleo \\
\hline \multirow{6}{*}{ Spilogale gracilis } & Acné & Carne, orina & Se consume la carne \\
\hline & Manchas en la piel & Carne & Se consume la carne \\
\hline & Bronquitis & Carne, orina & Sin especificar modo de uso \\
\hline & Limpieza de pulmones & Carne & Sin especificar modo de uso \\
\hline & Asma & Carne & Sin especificar modo de uso \\
\hline & Frio & Grasa & Sin especificar modo de uso \\
\hline \multirow{6}{*}{ Dasypus novemcinctus } & Tosferina o tos ahogona & Caparazón & Se pone a hervir en agua y se toma como agua de uso \\
\hline & $\begin{array}{l}\text { Dolor e inflamación mus- } \\
\text { cular }\end{array}$ & Grasa & Se coloca en las áreas afectadas \\
\hline & Bronquitis & Grasa, caparazón & Sin especificar modo de uso \\
\hline & Asma & Carne & Sin especificar modo de uso \\
\hline & Frio & Grasa & Sin especificar modo de uso \\
\hline & Planta de pie & Caparazón & Sin especificar modo de uso \\
\hline \multirow{6}{*}{ Nasua narica } & Calor en riñones & Grasa y animal completo & $\begin{array}{l}\text { La grasa del animal se unta sobre los riñones o la } \\
\text { persona enferma se debe sentar directamente sobre } \\
\text { el animal recién cazado }\end{array}$ \\
\hline & Dolores musculares & Grasa & Se aplica en áreas afectadas \\
\hline & Caídas & Grasa & Sin especificar modo de uso \\
\hline & Frio & Grasa & Sin especificar modo de uso \\
\hline & Dolor de cadera & Grasa & Sin especificar modo de uso \\
\hline & Inflamación & Grasa & Sin especificar modo de uso \\
\hline \multirow{2}{*}{ Didelphis virginiana } & Cintura & Cola & Sin especificar modo de uso \\
\hline & Empacho & Cola & Sin especificar modo de uso \\
\hline \multirow{6}{*}{ Canis latrans } & Dolores musculares & Grasa & Se coloca en áreas afectadas \\
\hline & Mal de ojo & Colmillos & $\begin{array}{l}\text { Son colocados en collares a los niños para prevenir } \\
\text { el "mal de ojo" }\end{array}$ \\
\hline & Tisis & Carne & Se consume la carne frita \\
\hline & Ataques & Carne & Sin especificar modo de uso \\
\hline & Reumas & Grasa & Sin especificar modo de uso \\
\hline & Frio & Grasa & Sin especificar modo de uso \\
\hline Urocyon cinereoargenteus & Sin especificar & Sin especificar & Sin especificar modo de uso \\
\hline \multirow{5}{*}{ Ctenosaura pectinata } & Debilidad por anemia & Sangre, carne & $\begin{array}{l}\text { Se consume la carne y la sangre para el tratamiento de } \\
\text { la anemia en niños y mujeres embarazadas }\end{array}$ \\
\hline & $\begin{array}{l}\text { "Reponer" a las embara- } \\
\text { zadas }\end{array}$ & Sangre & $\begin{array}{l}\text { Las mujeres embarazadas deben ingerir la sangre para } \\
\text { mantener la salud en dicha etapa }\end{array}$ \\
\hline & Vista cansada & Sangre, carne & La sangre y carne se ingieren para mejorar la vista \\
\hline & Quitar lo chipil a niños & Sangre & Sin especificar modo de uso \\
\hline & Acné & Sangre & Sin especificar modo de uso \\
\hline \multirow{3}{*}{ Kinosternon integrum } & Raquitismo infantil & Sangre & Se coloca sobre las coyunturas de los niños \\
\hline & Quitar lo chipil a niños & Animal completo y sangre & $\begin{array}{l}\text { El animal se coloca sobre la espalda de los infantes } \\
\text { para que camine sobre ellos }\end{array}$ \\
\hline & Para niños héticos & Animal completo & Sin especificar modo de uso \\
\hline \multirow{5}{*}{ Crotalus culminatus } & Cáncer & Carne & $\begin{array}{l}\text { El consumo de carne es para el tratamiento de casos de } \\
\text { cáncer en etapas iniciales o como prevención }\end{array}$ \\
\hline & Cáncer & Hiel & $\begin{array}{l}\text { Se consume la hiel para el tratamiento de cáncer en } \\
\text { etapas avanzadas }\end{array}$ \\
\hline & Picadura de alacrán & Piel y cascabel & $\begin{array}{l}\text { Un pedazo de la piel es aplicado directamente en la } \\
\text { zona de la picadura o se le agrega alcohol. El cascabel } \\
\text { se mezcla con vaporub aplicándose en el área afectada }\end{array}$ \\
\hline & Sangrado de nariz & Piel & $\begin{array}{l}\text { Un pedazo de piel con alcohol se coloca en la frente } \\
\text { para detener el sangrado }\end{array}$ \\
\hline & Gallinas tristes & Piel & La piel molida se agrega al agua que beben las gallinas \\
\hline \multirow{2}{*}{ Coragyps atratus, Cathartes aura } & Cáncer & Carne & Se consume la carne \\
\hline & Granos & Carne & Se consume la carne \\
\hline \multirow{2}{*}{ Geococcyx velox } & Diabetes & Carne & Sin especificar modo de uso \\
\hline & Aire & Carne & Sin especificar modo de uso \\
\hline Melanerpes chrysogenys & Padecimientos del corazón & Animal completo & Sin especificar modo de uso \\
\hline Agalychnis dacnicolor & Granos en la piel & Animal completo & $\begin{array}{l}\text { El animal completo se usa para masajear en el área } \\
\text { afectada }\end{array}$ \\
\hline Rhinella marina & Granos en la piel & Animal completo & $\begin{array}{l}\text { El animal completo se usa para masajear en el área } \\
\text { afectada }\end{array}$ \\
\hline
\end{tabular}


Otros valores de la fauna. - Los cazadores de subsistencia de áreas rurales utilizan de manera integral a los animales silvestres para obtener productos ornamentales o rituales, además de tenerlos como mascota e incluso conseguir algunas herramientas (Naranjo et al. 2004), tal como se registra en la región. Las estructuras como pieles, astas, patas, cabezas y caparazones son utilizadas para la elaboración de adornos para los hogares. La cabeza de venado se utiliza para elaborar trofeos de caza y colgarlos en la pared de los hogares de los cazadores. Otras estructuras como extremidades o colmillos, en un contexto simbólico, sirven para elaborar amuletos para atraer la buena suerte, como el caso de Didelphis virginiana, en cuyas patas se les amarra un listón rojo, se le pone una moneda y se mantiene como amuleto para la buena suerte. Otro uso del venado cola blanca es proveer de materia prima para la elaboración de herramientas. Las astas pequeñas se utilizan como agujas para componer las monturas de los caballos, las cabezas de venado con astas ramificadas se utilizan para la elaboración de percheros, mientras que su piel se puede emplear como tapetes o para elaborar correas. Sus extremidades inferiores se utilizan para la elaboración de fuetes o cuartas para los caballos y elaboración de percheros. Otra forma de uso de la fauna en la región es como animales de compañía. Por ejemplo, se capturan crías o huevos de aves silvestres como Ortalis poliocephala para empollarlos con gallinas. También en los hogares se mantienen individuos de Nasua narica, O. virginianus, Columbina spp., Anas spp. y Philortyx fasciatus.

Conflictos humano-fauna. - Las especies de fauna silvestre consideradas como dañinas son percibidas así porque provocan daños a actividades agrícolas, pecuarias, pesqueras o porque representan peligro para las personas. Es necesario precisar que no en todos los casos se termina con la muerte de los animales considerados perjudiciales, porque se emplean distintas medidas de mitigación o disminución de daños principalmente en actividades agrícolas como la vigilancia de los cultivos, colocación de espantapájaros, cuidado por perros o fumigar. En Ajuchitlán una medida de manejo era sembrar parcelas alrededor de las áreas agrícolas para que la fauna se alimentara de ellas, sin embargo, se dejaron de utilizar por falta de financiamiento (Sánchez 2019).

En la REBIOSH se ha registrado que la percepción de los daños a cultivos de maíz por la vida silvestre es alta (Flores-Armillas et al. 2020). El tejón (Nasua narica) es considerado como uno los animales que genera mayor impacto por la pérdida de cultivos de maíz o sorgo (Velarde \& Cruz 2015, Juárez-Mondragón 2017, Sánchez 2019) por sus hábitos alimentarios, alta tasa reproductiva, comportamiento gregario y abundancia. En las comunidades de El Limón, Ajuchitlán y Huautla se registró que los daños ocasionados por dicha especie y el venado cola blanca están relacionados con la distancia de las áreas agrícolas de los asentamientos humanos y la presencia de parches de vegetación en el área circundante, al ser más dañadas por la fauna aquellas parcelas alejadas de los pueblos y cerca de áreas boscosas (Flores-Armillas et al. 2020). En otros estudios también se registra al tejón como uno de los principales vertebrados silvestres que causan daños en la producción de maíz principalmente por su comportamiento gregario como se reporta en la Selva Lacandona, Chiapas (Romero-Balderas et al. 2006). Los carnívoros y rapaces como zorras, coyotes, tlacuaches, zorrillos, quebrantahuesos, gavilanes, aguilillas y cacomixtles son considerados como perjudiciales porque disminuyen la producción de aves de corral. Otro motivo importante para la cacería de especies perjudiciales es la disminución de accidentes ofídicos en áreas agrícolas y hogares, como en el caso de la Crotalus culminatus debido a que es un animal venenoso (Velarde \& Cruz, 2015), además de que los habitantes reconocen que tienen centros de salud sin personal de tiempo completo, no tienen acceso a antivenenos y para recibir atención se tienen que trasladarse a las cabeceras municipales en un contexto de servicio de transporte deficiente (Sánchez 2019).

La apropiación de fauna como una práctica multidimensional. - El aporte de la apropiación de fauna va más allá de la subsistencia material. La cacería de especies como el conejo (Sylvilagus spp.), iguana (Ctenosaura pectinata), venado cola blanca (O. virginianus), tórtolas (Columbia spp.), huilota (Zenaida spp.), chachalaca (Ortalis poliocephala) y codorniz (Philortyx fasciatus) es realizada con fines recreativos, lúdicos o de diversión (Velarde \& Cruz 2015, Juárez-Mondragón et al. 2017). Así mismo se realiza la preparación de carne de venado en eventos especiales para recibir personajes ajenos a las comunidades como presidentes municipales o servidores públicos de programas gubernamentales. El regalo de animales apropiados o bienes obtenidos de ellos es concebido en algunas comunidades de la región como una actividad que refuerza amistades o permite cerrar tratos (Velarde \& Cruz 2015). Desde un enfoque antropológico, las expresiones de solidaridad practicadas en la reciprocidad campesina, incluyen la ayuda mutua o trabajo colectivo, la redistribución de los medios de producción y los recursos (Sabourin 2001), como en este caso la fauna silvestre.

La apropiación de fauna también permite crear espacios de socialización, esparcimiento, convivencia y cohesión intra y extracomunitaria. Por ejemplo, la preparación de carne de venado para reuniones comunitarias; o la invitación para arreadas de venado a amistades de otras comunidades de Morelos, similar a lo que se describe en comunidades mayas del norte de Yucatán, donde dicha forma colectiva de apropiación representa un espacio de convivencia y socialización entre campesinos (Montiel et al. 2000).

En una perspectiva general, la apropiación de fauna silvestre como una práctica sociocultural tiene una función multidimensional, porque no solo aporta bienes materiales para la subsistencia, ya que alrededor de ella se desarrollan relaciones sociales, espacios que ayudan a la cohesión comunitaria y abarcan aspectos pocos considerados en el bienestar social de las comunidades rurales como las actividades de recreación y esparcimiento comunitario (Velarde \& Cruz 2015, Plata et al. 2019). 


\section{Conclusiones y perspectivas}

La apropiación de fauna silvestre es una práctica con arraigo cultural en comunidades de la Reserva de la Biosfera Sierra de Huautla. Esta práctica se realiza mediante la pesca, la recolección y principalmente la cacería. Para la extracción de los animales silvestres se utilizan diversos instrumentos como armas de fuego, perros, machetes, resorteras, nudos corredizos o se capturan presas manualmente. Dicha actividad puede ser fortuita $\mathrm{u}$ organizada al utilizar diferentes técnicas de apropiación como la arreada, la espiada o lampareada, las cuales muestran los conocimientos tradicionales que los habitantes poseen sobre su territorio y la fauna. Tradicionalmente, las especies animales silvestres proveen productos alimentarios, medicinales, ornamentales, así como animales de compañía, materiales para la fabricación de herramientas e incluso obtener recursos económicos. En la dinámica de apropiación, ciertas especies de fauna silvestre son percibidas como dañinas por los daños que ocasionan en las actividades agrícolas, pecuarias, pesqueras o porque representan peligro para las personas. La importancia de la apropiación está más allá del aporte para la subsistencia material para las comunidades locales, porque alrededor de esta se forman espacios de socialización, esparcimiento y cohesión comunitaria.

En una perspectiva general, el estudio de la relación sociedad-fauna ha recibido poca atención en la región, tanto del sector gubernamental como de la academia, resultando en pocos estudios. Para precisar la relevancia de la apropiación de fauna en la REBIOSH es necesario abordarla integralmente considerando aspectos socioculturales, ecológicos y económicos que permitan generar información para el diseño de estrategias de manejo para la conservación y el bienestar comunitario. En una perspectiva general latinoamericana, la investigación de la apropiación de fauna silvestre es relevante en contextos como en Sierra de Huautla donde habitan pueblos campesinos con tradición mesoamericana. La investigación para estos grupos es menor en comparación con comunidades originarias, a pesar de representar el grupo social con mayor número de personas que aprovechan a los animales silvestres en esta región del mundo (Pitriello \& Stronza 2019). Para los tomadores de decisiones, autoridades ambientales y diferentes grupos científicos, la apropiación de fauna silvestre es relacionada ipso facto con una actividad a erradicar, que debería prohibirse e incluso con poca importancia para la subsistencia y economía de las familias en el entorno rural. Sin embargo, es una práctica que forma parte de su realidad social, cultura y que ha encajado en la dinámica de sus formas de vida (Fa et al. 2013).

La información generada por los trabajos de investigación hasta ahora realizados en la REBIOSH, han sido principalmente de enfoque cualitativo, por lo que es necesario realizar aproximaciones cuantitativas de la apropiación de fauna. Para este fin, es necesario evaluarla de manera integral para la toma de decisiones, donde se consideren aspectos ecológicos como especies mayormente apropiadas, biomasa extraída, estado de las poblaciones de fauna, áreas potenciales de apropiación y económicos como el aporte de la fauna a la economía familiar.

Es preciso generar información sobre la contribución de los animales silvestres en la dieta familiar, lo que permitirá enmarcar su papel en la seguridad alimentaria. Este es un aspecto relevante para el diseño de estrategias de manejo sostenible, por ejemplo, en aquellas especies culturalmente importantes y en categoría de riesgo en la legislación mexicana como Ctenosaura pectinata.

Además, es importante considerar como una oportunidad para la conservación aspectos como la organización comunitaria y la cooperación entre localidades, al ser la fauna un recurso de uso común que se desplaza por diversas superficies ejidales en la REBIOSH. Esto con el propósito de fomentar el dialogo de saberes entre la población local, la academia y las autoridades gubernamentales, para promover espacios de participación social en la toma de decisiones sobre los recursos del territorio y establecer esquemas horizontales de manejo participativo.

\section{Literatura citada}

Abad-Fitz I, Maldonado B, Aguilar KM, Sánchez L, Gómez L, Casas A, Blancas J, García YM, Beltrán L, Sierra JA, Cristians S, Moreno AI, Torres I, Espinosa FJ. 2020. Consequences of Traditional Management in the Production and Quality of Copal Resin (Bursera bipinnata (Moc. \& Sessé ex DC.) Engl.) in Mexico. Forests 11(991).

Alarcón-Chaires P, Toledo VM. 2003. La etnoecología. [accessed 2021 Ene 30]. http://patrimoniobiocultural.com/archivos/publicaciones/articulos/NODO MICHOACAN TRIPTICO LA ETNOECOLOGIA.pdf

Alonso-Castro A. 2014. Use of medicinal fauna in Mexican traditional medicine. Journal of Ethnopharmacology 152:53-70. https://doi.org/10.1016/j. jep.2014.01.005

Alves RRN, Albuquerque U. 2018. Ethnozoology: Animals In Our Lives. Cambridge, Massachusetts: Academic Press. https://doi.org/10.1016/C2015-0-06858-7

Alves RRN, Alves HN. 2011. The faunal drugstore: Animal-based remedies used in traditional medicines in Latin America. Journal of Ethnobiology and Ethnomedicine 7(9). https://doi.org/10.1186/1746-4269-7-9

Argueta-Villamar A. 2008. Los saberes P'urhépecha. Los animales y el diálogo con la naturaleza. Morelia, México: Universidad Nacional Autónoma de México, Universidad Michoacana de San Nicolás de Hidalgo, Programa de las Naciones Unidas para el Medio Ambiente, Gobierno del Estado de Michoacán y Universidad Intercultural.

Arias D, Barona C, Dorado O. 2014. Una mirada a la biodiversidad y conservación de Morelos desde un enfoque educativo. Cuernavaca, México: Universidad Autónoma del Estado de Morelos.

Ávila-Nájera DM, David-Mendoza G, Villarreal O, Serna-Lagunes R. 2018b. Uso y valor cultural de la herpetofauna en México: una revisión de las últimas dos décadas (19972017). Acta Zoológica Mexicana nueva serie 34(1):115. https://doi.org/10.21829/azm.2018.3412126

Ávila-Nájera DM, Naranjo EJ, Tigar BJ, Villarreal OA, Mendoza GD. 2018a. An Evaluation of the Contemporary Uses and Cultural Significance of Mammals in Mexi- 
co. Ethnobiology Letters 9(2):124-135. https://doi. org/10.14237/ebl.9.2.2018.1106

Bello M. 2015. Uso tradicional de la fauna silvestre en la comunidad el Zoquital (Amacuzac) en la Reserva de la Biósfera Sierra de Huautla, Morelos. Tesis de Licenciatura. Universidad Autónoma del Estado de Morelos, Cuernavaca, México.

Burgos-Herrera B, Saldaña-Fernández MC, López-Medellín X. 2019. Importancia cultural de especies forestales útiles, en tres comunidades de la Sierra de Huautla, Morelos, México. Ambiente y Desarrollo 23(45). https:// doi.org/10.11144/Javeriana.ayd23-45.icef

Bustos-Zagal G, Guzmán-Ramírez R, Castro-Franco R, GarcíaFlores A, Trujillo-Jiménez P. 2019. Ecology and Use of Iguanas Ctenosaura pectinata (Sauria: Iguanidae) in Two Rural Communities in Morelos, Mexico. Journal of Zoological Sciences 7:5-12.

Cano E, Medinaceli A, Sanabria O, Argueta A. 2014. Código de Ética para la investigación, la investigación-acción y la colaboración etnocientífica en América Latina. México: Asociación Etnobiologíca Mexicana, Sociedad Latinoamericana de Etnobiología.

CONABIO (Comisión Nacional para el Conocimiento y Uso de la Biodiversidad), UAEM (Universidad Autónoma del Estado de Morelos). 2004. La Diversidad Biológica en Morelos: Estudio del Estado. México: Comisión Nacional para el Conocimiento y Uso de la Biodiversidad, Universidad Autónoma del Estado de Morelos.

CONABIO (Comisión Nacional para el Conocimiento y Uso de la Biodiversidad). 2020. Animales medicinales. [accessed $2021 \mathrm{Abr} 12$ ]. https://www.biodiversidad.gob. $\mathrm{mx} /$ diversidad/medicinal/animales

CONANP (Comisión Nacional de Áreas Naturales Protegidas). 2005. Programa de conservación y manejo de la Reserva de la Biosfera Sierra de Huautla. Ciudad de México: Comisión Nacional de Áreas Naturales Protegidas.

Cruz-Aguilar R, Cruz-León A, Cuevas-Reyes V, Ramírez-Valverde B. 2018. Impacto social de la mercantilización de la naturaleza en la Sierra de Huautla, Morelos. Estudios sociales 28 (51):1-23. https://doi.org/10.24836/ es.v28i51.521

Cruz-Aguilar R. 2018. Mercantilización de la naturaleza, saberes tradicionales y desarrollo rural alternativo en la Sierra de Huautla, Morelos. Tesis de Doctorado. Universidad Autónoma Chapingo, Texcoco, México.

Cruz-León A, Uribe-Gómez M, Lara-Bueno A, Yescas-Albarrán C, Maldonado-Torres R. 2016. Diálogo del saber campesino y la investigación científica: árboles nativos dendroenergéticos en la Reserva de la biosfera Sierra de Huautla, Morelos, México. Revue d'ethnoécologie 9:1-14.

Cruz-León A. 2008. Frutales de recolección en la Sierra de Huautla, Morelos. En: Cruz J, Torres P. (eds), Enfoques tecnológicos en la fruticultura: un tributo a Raúl Mosqueda. Universidad Autónoma Chapingo, Ciudad de México. Pp. 117-129.

De Sahagún, B. 2005. Fauna de Nueva España. Ciudad de México: Fondo de Cultura Económica.

DOF (Diario Oficial de la Federación). 1999. [accessed $2021 \mathrm{Abr}$ 10]. http://dof.gob.mx/nota detalle.php?codigo=495 3998\&fecha $=15 / 09 / 1999$

DOF (Diario Oficial de la Federación). 2010. NORMA Oficial Mexicana NOM-059-SEMARNAT-2010, Protección ambiental-Especies nativas de México de flora y fauna silvestres-Categorías de riesgo y especificaciones para su inclusión, exclusión o cambio-Lista de especies en riesgo. [accessed 2021 Abr 10]. https://www.profepa. gob.mx/innovaportal/file/435/1/NOM 059 SEMARNAT 2010.pdf

DOF (Diario Oficial de la Federación). 2018. Ley General de Vida Silvestre. [accessed 2021 Abr 10]. http://www. diputados.gob.mx/LeyesBiblio/pdf/146_190118.pdf

Domínguez E. 2015. Evaluación de la explotación pesquera de los bagres Ictalurus balsanus e Ictalurus puntactus y propuesta de manejo en la comunidad de Xicatlacotla, Tlaquiltenango, Morelos. Tesis de Maestría. Universidad Autónoma del Estado de Morelos, Cuernavaca, México.

Dorado O, Arias D, Alonso G, Maldonado B. 2002. Educación ambiental para la biodiversidad en el trópico seco, Reserva de la Biosfera Sierra de Huautla, Morelos, México. Tópicos en Educación Ambiental 4:31.

Durand L. 2010. Pensar positivo no basta. Actitudes en torno a la conservación en la Reserva de la Biosfera Sierra de Huautla, México. Interciencia 35:430-436.

Estrada-Portillo DS, Rosas-Rosas OC, Parra-Inzunza F, Guerrero-Rodríguez JD, Tarango-Arámbula LA. 2018. Valor de uso, importancia cultural y percepciones sobre mamíferos silvestres medianos y grandes en la mixteca poblana. Acta Zoológica Mexicana nueva serie 34:115. https://doi.org/10.21829/azm.2018.3412131

Fa JE, Farfán MA, Márquez AL, Duarte J, Vargas JM. 2013. Reflexiones sobre el impacto y manejo de la caza de mamíferos silvestres en los bosques tropicales. Ecosistemas 22(2):76-83. https://doi.org/10.7818/ ECOS.2013.22-2.12

Flores-Armillas V, López-Medellín X, García R, MacGregor I, Valenzuela D. 2020. Landscape Features Associated with Damage to Maize (Zea mays) Fields in Central México: A Comparison of Wind and Wildlife Damage. Agriculture 10, 460. https://doi.org/10.3390/agriculture 10100460

Gallina-Tessaro S, Hernández-Huerta A, Delfín-Alfonso C, González-Gallina A. 2009. Unidades para la conservación, manejo y aprovechamiento sustentable de la vida silvestre en México (UMA). Retos para su correcto funcionamiento. Investigación Ambiental 1:143-152.

García-Flores A, Lozano-García MA, Ortiz-Villaseñor AL, Monroy-Martínez R. 2014. Uso de mamíferos silvestres por habitantes del Parque Nacional El Tepozteco, Morelos, México. Etnobiología 12:57-67.

García-Flores A, Valle-Marquina R, Monroy-Martínez R. 2018. Aprovechamiento tradicional de mamíferos silvestres en Pitzotlán, Morelos, México. Revista Colombiana De Ciencia Animal-RECIA 10:111-123. https://doi. org/10.24188/recia.v10.n2.2018.620

García-Flores A. 2008. La etnozoología como una alternativa para el desarrollo comunitario sustentable en la Reserva Estatal Sierra de Monte Negro, Morelos, México. Tesis de Maestría. Universidad Autónoma del Estado de Morelos, Cuernavaca, México.

González-Rebeles I, Méndez M, Anaya L, Juárez-Mondragón A. 2020. Unidades de manejo para la conservación de vida silvestre. En: Comisión Nacional para el Conocimiento y Uso de la Biodiversidad, Universidad Autónoma del Estado de Morelos, Secretaria de Desarrollo Sustentable, Comisión Estatal de Biodiversidad. (eds), La biodiversidad del estado de Morelos. Estudio de estado 2. Vol. 1. Comisión Nacional para el Conocimiento y Uso de la Biodiversidad, Cuidad de México. Pp. 333345. 
Götz C. 2014. La alimentación de los mayas prehispánicos vista desde la zooarqueología. Anales de Antropología 48(1):167-199. https://doi.org/10.1016/S01851225(14)70494-1

Guerra RM, Calme S, Gallina TS, Naranjo PE. 2010. Uso y manejo de fauna silvestre en el norte de Mesoamérica. México: Instituto Nacional de Ecología.

Hernández-Tapia R, Valverde T, Aranda A, Martínez-Peralta C, Platas-Neri D. 2018. Traditional Knowledge as a Basis for the Development of a Sustainable Resource Management Program: A Case Study in a Rural Village in Morelos, Mexico. Ethnobiology Letters 9:1-11. https://doi.org/10.14237/ebl.9.2.2018.870

Herrera-Flores B, Santos-Fita D, Naranjo E, Hernández-Betancourt S. 2019. Importancia cultural de la fauna silvestre en comunidades rurales del norte de Yucatán, México. Península 14:27-55.

INEGI (Instituto Nacional de Estadística y Geografía). 2010. Censo de población. México: Instituto Nacional de Estadística y Geografía.

Juárez-Mondragón A, González-Rebeles C, Castillo A, GarcíaFrapolli E, Ordoñez-Díaz M. 2015. La vida silvestre manejada como recursos de uso común: estudio de caso en México. Tropical and Subtropical Agroecosystems 18:313-331.

Juárez-Mondragón A. 2017. Prácticas de aprovechamiento en Unidades de Manejo para la Conservación de Vida Silvestre (UMA) del sur de Morelos. Tesis de Maestría. Universidad Nacional Autónoma de México, Ciudad de México, México.

Juárez-Mondragón A. 2019. Prácticas de aprovechamiento en Unidades de Manejo para la Conservación de la Vida Silvestre (UMA) del sur de Morelos. México: Universidad Nacional Autónoma de México.

López CC, Zazueta AX, Porras AC. 2005. Notas sobre el aprovechamiento del medio ambiente nortense: reconocimiento del terreno, inicio de la cacería. Ra Ximhai 1(1):39-50.

López-Medellín X, Vázquez LB, Valenzuela-Galván D, Wehncke E, Maldonado B, Durand-Smith L. 2017. Percepciones de los habitantes de la Reserva de la Biósfera Sierra de Huautla: hacia el desarrollo de nuevas estrategias de manejo participativo. Interciencia 42(1):8-16.

Maldonado BJ. 1997. Aprovechamiento de los recursos florísticos de la Sierra de Huautla Morelos, México. Tesis de Maestría. Universidad Nacional Autónoma de México, Ciudad de México, México.

Maldonado BJ. 2013. Patrones de uso y manejo de los recursos florísticos del bosque tropical caducifolio en la Cuenca del Balsas, México. Tesis de Doctorado. Universidad Nacional Autónoma de México, Ciudad de México, México.

Medrano C. 2012. Etnozoología, usos y abusos de los cuestionarios. Papeles de Trabajo Centro de Estudios Interdisciplinarios en Etnolingüística y Antropología SocioCultural 23: 59-81.

Méndez-Sánchez J. 2020. El cazador, la presa y el Dueño de los Animales: El complejo cinegético nahua. Trace 78:4366. http://dx.doi.org/10.22134/trace.78.2020.738

Monroy R, Pino J, Lozano M, García-Flores A. 2011. Estudio etnomastozoológico en el Corredor Biológico Chichinautzin (COBIO), Morelos. Sitientibus série Ciências Biológicas 11:16-23. http://dx.doi.org/10.13102/ $\underline{\operatorname{scb} 101}$

Monroy-Vilchis O, Cabrera L, Suárez P, Zarco-González M, Ro-
dríguez-Soto C, Urios V. 2008. Uso tradicional de vertebrados silvestres en la sierra Nanchititla, México. Interciencia 33:308-313.

Montiel S, Arias L, Dickinson F. 2000. La cacería tradicional en el norte de Yucatán: una práctica comunitaria. Revista de Geografía Agrícola 29:43-52.

Naranjo EJ, Cuarón AD. 2010. Usos de la fauna silvestre. En: Ceballos G, Martínez L, García A, Espinoza E, Bezaury J, Dirzo R. (eds), Diversidad, amenazas y áreas prioritarias para la conservación de las selvas secas del Pacífico de México. Fondo de Cultura Económica y Comisión Nacional para el Conocimiento y Uso de la Biodiversidad, Ciudad de México. Pp. 271-284.

Naranjo EJ, Guerra MM, Bodmer RE, Bolaños JE. 2004. Subsistence hunting by three ethnic groups of the Lacandon Forest, Mexico. Journal of Ethnobiology 24: 233-253.

Ojasti J, Dallmeier F. 2000. Manejo de Fauna Silvestre Neotropical. Washington: Smithsonian Institution/MAB Biodiversity Program.

Olivier G. 2015. Cacería, sacrificio y poder en Mesoamérica. Tras las huellas de Mixcóatl, "Serpiente de Nube". México: Fondo de Cultura Económica, Instituto de Investigaciones Históricas-Universidad Nacional Autónoma de México, Centro de Estudios Mexicanos y Centroamericanos

Palacios A. 2012. Aprovechamiento de fauna silvestre en Nexpa y San José de Pala, Tlaquiltenango, Morelos. Tesis de Maestría. Universidad Autónoma del Estado de Morelos, Cuernavaca, México.

Paredes-Lira E. 2013. Aspectos socioeconómicos de la pesquería de bagre Ictalurus balsanus en el rio Amacuzac, Morelos. Tesis de Maestría. Universidad Autónoma del Estado de Morelos, Cuernavaca, México.

Pérez-Gil R, Jaramillo F, Muñiz AM, Torres MG. 1995. Importancia económica de los vertebrados silvestres en México. México: PG-7 Consultores SC, CONABIO.

Pérez-Soto M, Alpuche 0, Pérez F. 2016. Situación actual de las unidades de manejo ambiental en la Reserva de la Biosfera de la Sierra de Huautla, Morelos. [accessed 2021 Abr 20].https://www.ecorfan.org/handbooks/ Handbook Produccion Comercializacion y Medio Ambiente T1V1/Particiones/2.pdf

Pérez-Soto MT. 2016. Las necesidades básicas insatisfechas en las unidades de manejo ambiental en el estado de Morelos. Perspectivas Rurales 14:75-91.

Pitriello M, Stronza A. 2019. Campesino hunting and conservation in Latin America. Conservation Biology 0(0):1-16. https://doi.org/10.1111/cobi.13396

Plata E, Montiel S, Fraga J, Evia C. 2019. Sociocultural Importance of Dogs (Canis lupus familiaris) in Maya Subsistence Hunting: Revelations From Their Participation in the Traditional Group Hunting (Batida) in Yucatan. Tropical Conservation Science 12:1-11. https://doi. org/10.1177\%2F1940082919830829

Ramírez-Barajas J, Calme S. 2015. Subsistence Hunting and Conservation. En: Islebe G, Calme S, Leon J, Schmook B. (eds), Biodiversity and Conservation of the Yucatán Peninsula. Springer International Publishing, New York. Pp. 333-354.

Romero-Balderas K, Naranjo E, Morales H, Nigh R. 2006. Daños ocasionados por vertebrados silvestres al cultivo de maíz en la selva lacandona, Chiapas, México. Interciencia 31:276-283.

Romero-Navarrete L. 2015. El ejido mexicano: entre la persis- 
tencia y la privatización. Argumentos 28(79): 217238.

Sabourin E. 2001. Reciprocidad e intercambio en comunidades campesinas del Nordeste: Massaroca (Bahía, Brasil). Revista Iberoamericana de Autogestión y Acción Comunal 35:101-112.

Saldaña-Fernández C. 2012. Delimitaciones territoriales para la conservación ambiental. Inventio 8(16):5-12.

Sánchez L. 2019. Interacción humano-fauna silvestre y el daño ocasionado a los cultivos en la Reserva de la Biósfera Sierra de Huautla: percepciones y actitudes. Tesis de Licenciatura. Universidad Autónoma del Estado de Morelos, Cuernavaca, México.

Santos-Fita D, Naranjo E, Rangel-Salazar L. 2012. Wildlife uses and hunting patterns in rural communities of the Yucatan Peninsula, Mexico. Journal of Ethnobiology and Ethomedicine 8:1-18. https://doi.org/10.1186/17464269-8-38

Santos-Fita D. 2018. Subsistence Hunting in Rural Communities: Incompatibilities and Opportunities within Mexican Environmental Legislation. Journal of Ethnobiology 38:356-371. https://doi.org/10.2993/0278$\underline{0771-38.3 .356}$

Solís L, Casas A. 2019. Cuicatec ethnozoology: traditional knowledge, use, and management of fauna by people of San Lorenzo Pápalo, Oaxaca, Mexico. Journal of Ethnobiology and Ethnomedicine 15,58. https://doi. org/10.1186/s13002-019-0340-1

Sorani V, Rodríguez G, Valenzuela D. 2020. Uso de suelo y conservación de la selva baja caducifolia en la Sierra de Huautla. En: Comisión Nacional para el Conocimiento y Uso de la Biodiversidad, Universidad Autónoma del Estado de Morelos, Secretaria de Desarrollo Sustentable, Comisión Estatal de Biodiversidad, eds. La biodiversidad del estado de Morelos. Estudio de estado 2. Vol. 1. Comisión Nacional para el Conocimiento y Uso de la Biodiversidad, Ciudad de México. Pp. 88-90.

Tejeda-Cruz C, Naranjo-Piñera E, Medina-Sansón L, GuevaraHernández F. 2014. Cacería de subsistencia en comunidades rurales de la selva Lacandona, Chiapas, México. Revista Quehacer Científico en Chiapas 9(1):59-73.

Toledo V, Barrera-Bassols N, Boege E. 2019. ¿Qué es la diversidad biocultural? México: Universidad Nacional Autónoma de México.

Toledo V. 2008. Metabolismos rurales: hacia una teoría económico-ecológica de la apropiación de la naturaleza. Revista Iberoamericana de Economía Ecológica 7:1-26.

Toledo-Hernández VH, Corona-López AM, Flores-Palacios A., Coronado-Blanco JM, Nikolavena-Myartseva S. 2012. Riqueza de coleópteros (Insecta: Coleoptera) de la Reserva de la Biosfera Sierra de Huautla, México. En: Ruiz-Cancino E, Coronado-Blanco J. (eds), Recursos naturales, Universidad Autónoma de Tamaulipas Ciudad Victoria, Tamaulipas. Pp.142-150.

Trujillo-Santisteban L, López-Medellín X. 2018. ¿Qué es la conservación desde el punto de vista de los campesinos? Condiciones productivas en un área natural protegida, Morelos, México. Etnobiología 16(1):58-72.

Valadez-Azúa R, Rodríguez-Galicia B. 2014. Uso de la fauna, estudios arqueozoológicos y tendencias alimentarias en culturas prehispánicas del centro de México. Anales de Antropología 48(1):139-166. https://doi. org/10.1016/S0185-1225(14)70493-X

Valenzuela D, Dorado O, Ramírez R. 2010. Sierra de Huautla, Morelos. Guerrero, Puebla. En: Ceballos G, Martínez L,
García A, Espinoza E, Bezaury J, Dirzo R. (eds), Diversidad, amenazas y áreas prioritarias para la conservación de las selvas secas del Pacífico de México, Fondo de Cultura Económica y Comisión Nacional para el Conocimiento y Uso de la Biodiversidad, Ciudad de México. Pp. 477-481.

Valle R. 2017. Aprovechamiento tradicional de mamíferos silvestres en Pitzotlán, Tepalcingo, Morelos. Tesis de Licenciatura. Universidad Autónoma del Estado de Morelos, Cuernavaca, México.

Velarde S, Cruz A. 2015. La fauna silvestre y su relación con el bienestar de tres comunidades de la Reserva de la Biosfera Sierra de Huautla, Morelos. Etnobiología $13: 39-52$

Velarde S. 2010. Aprovechamiento y usos tradicionales de fauna silvestre como factor de desarrollo regional en tres comunidades en la Reserva de la Biosfera Sierra de Huautla, Morelos. Tesis de Maestría. Universidad Autónoma Chapingo, Texcoco, México.

Yescas-Albarrán C, Cruz-León A, Uribe-Gómez M, Lara-Bueno A, Maldonado-Torres R. 2016. Árboles nativos con potencial dendroenergético para el diseño de tecnologías agroforestales en Tepalcingo, Morelos. Revista Mexicana de Ciencias Agrícolas 16:3301-3313.

Zarazúa-Carbajal M, Chávez-Gutiérrez M, Romero-Bautista Y, Rangel S, Moreno AI, Alvarado L, Smith S, Blancas J, De Val E, Coro M, Casas A. (2020). Use and management of wild fauna by people of the Tehuacán-Cuicatlán Valley and surrounding areas, Mexico. Journal of Ethnobiology and Ethnomedicine 16,4. https://doi.org/10.1186/ s13002-020-0354-8

\section{Agradecimientos / Acknowledgments:}

A los habitantes en Sierra de Huautla que nos compartieron su vida cotidiana.

Conflicto de intereses / Competing interests:

Los autores no incurren en conflictos de intereses.

Rol de los autores / Authors Roles:

RVM: Investigación, metodología, conceptualización, redacciónborrador original, redacción, revisión y edición. AGF: Administración del proyecto, supervisión, conceptualización, análisis formal, metodología, redacción-borrador original, redacción-revisión y edición. HCB: Análisis formal, metodología, redacción-borrador original, redacción-revisión y edición.

Fuentes de financiamiento / Funding:

Los autores declaran que esta investigación no recibió ninguna subvención específica de ninguna agencia de financiación, ni del sector comercial o sin fines de lucro.

\section{Aspectos éticos / legales; Ethics / legals:}

Los autores declaran no haber incurrido en aspectos no éticos. En el trabajo de campo se siguieron las pautas del Código de Ética de la Sociedad Latinoamericana de Etnobiología sobre la obtención del permiso de las autoridades locales, el principio del consentimiento libre e informado y el principio de confidencialidad. 